University of Louisville

ThinkIR: The University of Louisville's Institutional Repository

\title{
Interactions between human neutrophils and mycobacterium smegmatis : a comparative general analysis.
}

Irina Miralda

University of Louisville

Follow this and additional works at: https://ir.library.louisville.edu/honors

Part of the Biology Commons, and the Medical Sciences Commons

\section{Recommended Citation}

Miralda, Irina, "Interactions between human neutrophils and mycobacterium smegmatis : a comparative general analysis." (2014). College of Arts \& Sciences Senior Honors Theses. Paper 81.

http://doi.org/10.18297/honors/81

This Senior Honors Thesis is brought to you for free and open access by the College of Arts \& Sciences at ThinkIR: The University of Louisville's Institutional Repository. It has been accepted for inclusion in College of Arts \& Sciences Senior Honors Theses by an authorized administrator of ThinkIR: The University of Louisville's Institutional Repository. This title appears here courtesy of the author, who has retained all other copyrights. For more information, please contact thinkir@louisville.edu. 
Interactions between human neutrophils and Mycobacterium smegmatis: A comparative, general analysis

By

Irina Miralda

Submitted in partial fulfillment of the requirements for Graduation (summa or magna) cum laude

and

for Graduation with Honors from the Department of Biology (if pertinent)

University of Louisville

May, 2014 


\section{Abstract}

Mycobacteria smegmatis is an abundant soil and water inhabitant with which humans are continuously in contact. Despite the fact that it is typically considered non-pathogenic, a few rare cases of $M$. smegmatis-caused infections have been reported and it has been shown that $M$. smegmatis is able to modulate inflammatory responses in macrophages. Neutrophils are the innate immune system's first line of centralized defense against invading microbes, especially the frequently encountered M. smegmatis. However, very little information is known of the mechanism by which neutrophils eliminate environmental bacteria. In this study, the hypothesis that M. smegmatis regulates neutrophil functional responses, and that some of these manipulations may involve $M$. smegmatis' virulence-associated glycolipid, PILAM was tested by specifically examining its effect on two of the major neutrophil responses: cytokine release and bacterial killing through degranulation and respiratory burst activity. Our data showed that this bacterium promotes inflammation, tissue damage and manipulates the $T_{h} 1$ response by controlling IL-12. In addition, PILAM seems to be involved in the neutrophil responses of intracellular ROS, gelatinase granule exocytosis and IL-12 release. Consequently, the interaction between human neutrophils and M. smegmatis appears to be much more intricate than formerly described. 


\section{Table of Contents}

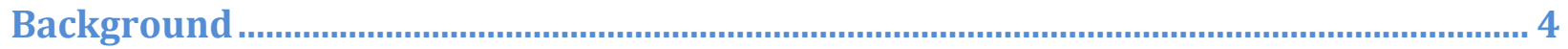

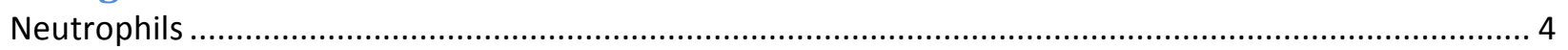

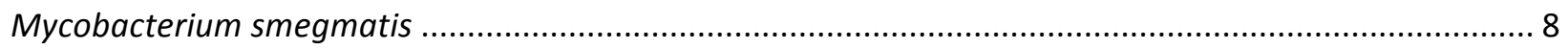

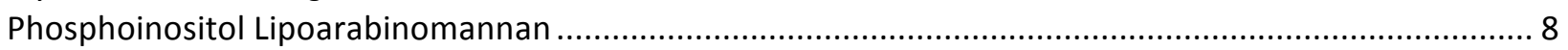

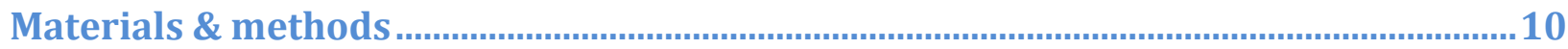

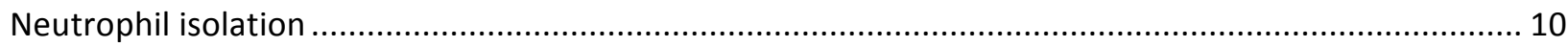

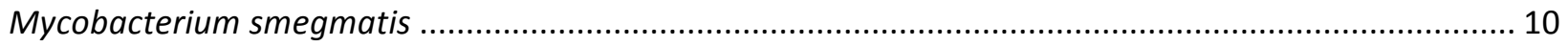

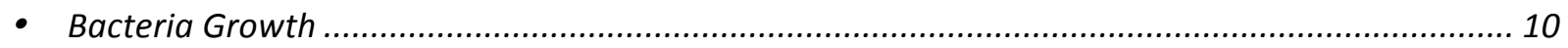

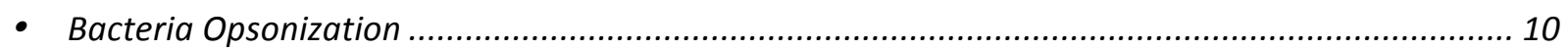

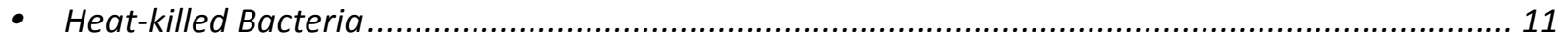

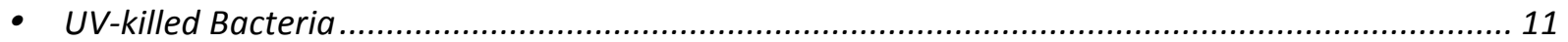

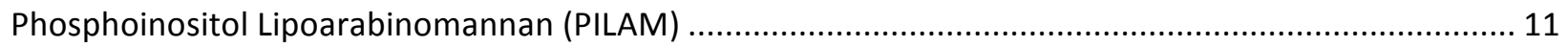

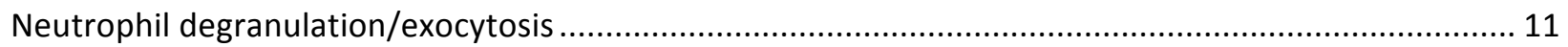

- Measurement of azurophil granules, specific granules, and secretory vesicles ............................ 11

-........ Measurement of degranulation of gelatinase granules using Enzyme-linked immunosorbent Assay

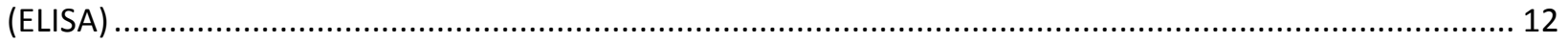

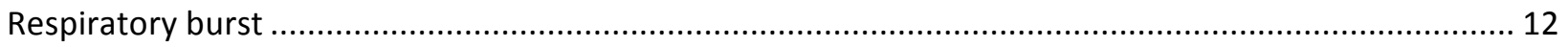

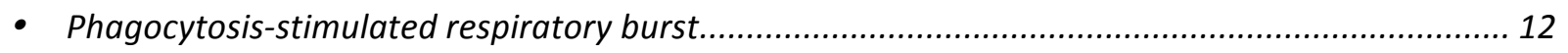

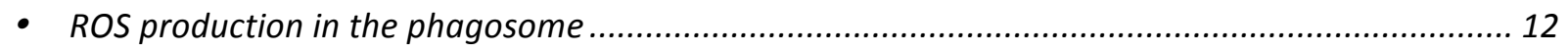

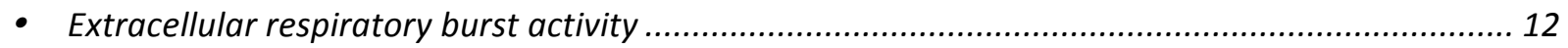

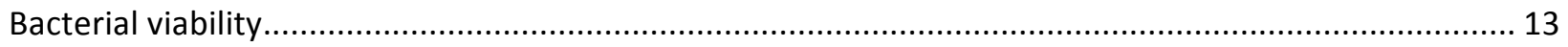

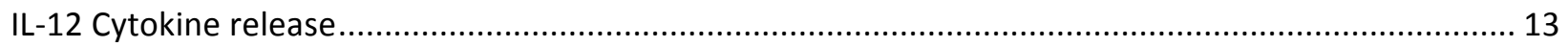

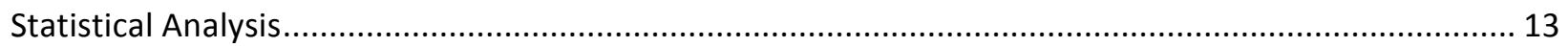

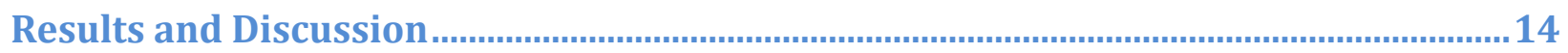

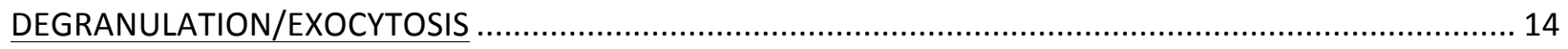

Neither PILAM nor M. smegmatis have an effect on secretory vesicle exocytosis .............................. 14

Non-opsonized M. smegmatis significantly induced gelatinase granule release. ................................ 15

Neither M. smegmatis nor PILAM induced specific granule exocytosis............................................ 16

Non-opsonized and UV-killed M. smegmatis significantly induced a time-dependent exocytosis of

azurophil granules while opsonized and PILAM inhibited degranulation......................................... 17

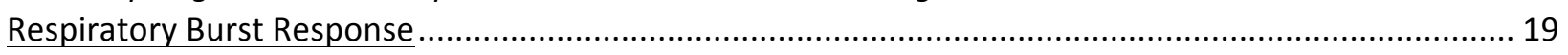

Opsonized M. smegmatis and PILAM significantly induce a time-dependent increase in intracellular

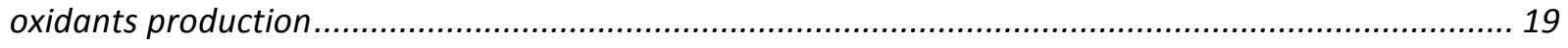

Intracellular oxidants accumulate within phagosomes that contain M. smegmatis........................... 21

Opsonized M. smegmatis induced a potent extracellular oxidative burst.......................................... 23

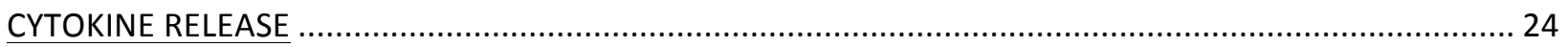

M. smegmatis reduced basal reserves of IL-12 without involvement by PILAM................................. 25

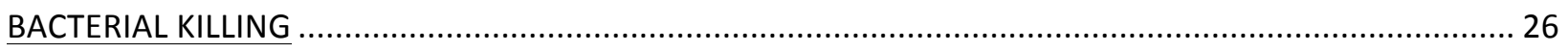

Neutrophils may or may not kill M. smegmatis at 30 minutes post-infection.................................... 26

Conclusions \& Future Directions .......... 28

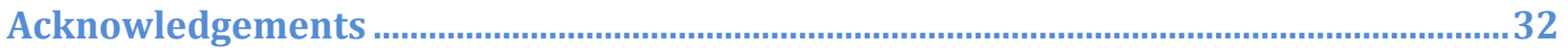

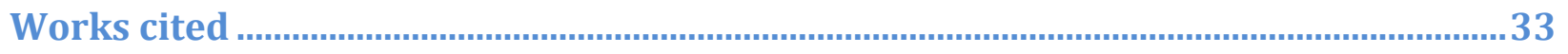




\section{Background}

Humans are in daily, continuous contact with an innumerable amount of bacteria; however, the majority of bacterial exposure does not lead to illness or disease. The first barriers against most microorganisms are the epithelial surfaces of the body, but if these surfaces are breached, the immune system will become involved to control or eliminate any foreign entities. The immune system is split into innate and adaptive immunity, each encompassing different but overlapping functions (1). Innate immunity is characterized by its built-in, non-specific and immediate responses for recognition and destruction of any foreign microorganism. On the other hand, the adaptive immunity presents an acquired, specific, memory-dependent protection against microorganisms to prevent reinfection by the same pathogens. The functional variability between the wings of the immune system is mediated through the cells associated with each wing; monocytes, macrophages, dendritic cells and polymorphonuclear cells make up the innate immune cell response whereas $B$ and $\mathrm{T}$ cell lymphocytes manage the adaptive immune response (1).

\section{Neutrophils}

Polymorphonuclear cells (PMNs), which are commonly known as neutrophils, are indispensible in the protection against infectious agents that have penetrated the physical barriers of the human body and play a large role in the development of innate and acute inflammatory responses. After being produced in great quantities in the bone marrow (2), these leukocytes are released into the blood stream in their naïve state where they scour the body for signs of infection and inflammation (3). The power of neutrophil involvement in host responses is most dramatically emphasized in individuals with neutrophil genetic disorders or low peripheral neutrophil counts. Without the critical quantity of neutrophils necessary to block microbial advances, infections develop with increased frequency, severity, and overall host mortality (4).

The recruitment of neutrophils to sites of infection begins when local macrophages produce signals called cytokines and chemokines in response to microbial intruders. These compounds activate the local endothelial cells of the vascular walls, which will 
subsequently express selectins and integrins on their membranes and capture and activate patrolling neutrophils (2). The activated neutrophils can then migrate into the infected tissue by either penetrating an individual endothelial cell or wedging between two endothelial cells. In the infected area, the products generated by live bacteria or other proteins produced by local immune cells act as chemotactic signals that will guide the neutrophils to the intruders (2). Once inside the area of infection, neutrophils can perform an array of sophisticated functions to control microbial pathogenesis. The two functions of most relevance to this project, which will be described in detail, are neutrophils' ability to destroy microbes through either intracellular or extracellular means and neutrophils' capacity to produce and release cytokines for recruitment and activation of other immune cells.

The best established role of neutrophils is that of professional phagocytic cells, whose microbial killing capabilities involve both oxygen dependent and independent mechanisms. Intracellular killing by neutrophils first involves the uptake of bacteria into a vacuole by a process called phagocytosis. The phagocytic event triggers activation of the NADPH oxidase multi-enzymatic complex, resulting in oxygen consumption with the generation of reactive oxygen species (ROS) called the respiratory burst. Depending on the stimuli, the newly formed reactive oxygen species can be unloaded into the phagosomal vacuole or released from the neutrophil. In addition, characteristic neutrophil granules fuse with the phagocytic vacuole, releasing their microbicidal protein content, and contributing to the maturation of the phagosome (5). Since these granules are filled with antimicrobial and tissue-digesting compounds, the combination of the reactive oxidative species and the digestive granule proteins creates a deadly cocktail (6). This intricate process begins with either direct recognition of the trespassing microbe by pattern recognition receptors (TLRs) and/or by phagocytic receptors such as Fc $\gamma$ Rs and complement receptors that are present on the neutrophil plasma membrane (7). Microbial opsonization is the process by which the complement system tags pathogens with serum components C1-C9. These serum components that opsonize bacteria are analogous to antibodies in the adaptive immune system because they mediate pathogen recognition and phagocytosis, but in a non-specific way. Studies have confirmed that human neutrophils 
can recognize and internalize both opsonized and non-opsonized bacteria, although bacteria uptake by neutrophils is more efficient with opsonized bacteria (8).

Neutrophil granules play a fundamental role in neutrophil microbial killing. The abundant neutrophil granules can be divided into four subtypes and make neutrophils part of the exclusive, 3-member granulocyte family (6). The four granule subtypes are formed at different stages of neutrophil maturation within the bone marrow and display great heterogeneity in their content as well as their readiness for exocytosis after stimulation. As the nature of the granule content becomes more destructive, the degranulation becomes increasingly difficult and highly controlled (9). This specificity of release gives a glimpse of the sophistication of neutrophils, given that the uncontrolled liberation of ROS and granule content would harm host tissues as an undesirable side effect.

Some of the granule content is found in several granule types; however, each granule subtype will have exclusive granule content and plasma membrane specific markers, which allows for quantification of each granule's activity. The most readily released granules, the secretory vesicles, are the last ones formed during neutrophil maturation. Although these vesicles only contain harmless plasma proteins within, the vesicle membrane is abundant in receptors. Because of their readiness in mobilization, it is believed that they are released when the neutrophil first encounters the endothelium that has become activated by chemokines and cytokines that indicate a bacterial presence (10). With the fusion of granules into the neutrophil's plasma membrane, all the receptors present on the vesicle membrane are incorporated into the cell's plasma membrane, transforming the neutrophils from a passive cell to a highly sensitive, primed one (11). Gelatinase or tertiary granules are the third granules formed during neutrophil maturation, and require a stronger stimulus to be mobilized compared to secretory vesicles. Gelatinase granules contain a high number of matrix metalloproteases (MMPs) such as MMP-9 (gelatinase) and MMP-8 (collagenase). These enzymes have protease activity and allow neutrophil migration into tissues from the endothelial lining via the breakdown of the basal membrane (2).

Specific or secondary granules are the second produced and require a stronger stimulus to be mobilized compared to gelatinase granules. Containing of high concentrations of lactoferrin, the specific granules are associated with bacteriostatic and 
bactericidal functions. Lactoferrin is a protein that impedes bacterial proliferation by sequestering iron from the surrounding environment and disrupting the outer bacterial membrane through the dispersion of components such as lipopolysaccharide (LPS) (12). Azurophil or primary granules are the first formed during neutrophil maturation and are the most difficult to mobilize, but also house some of the deadliest protein contents: myeloperoxidase and defensins $(9,10)$. The azurophil granules' myeloperoxidase kills and digests bacteria by targeting their membrane transport proteins, adenosine triphosphate (ATP) generating systems, and DNA replication origins (13). In addition, myeloperoxidase catalyzes the production of additional strong microbicidal compounds, like hypochlorous acid ( $\mathrm{HOCl}) . \mathrm{HOCl}$ is made when the superoxide from the respiratory burst undergo dismutation into hydrogen peroxide that is combined with chloride anions in the phagosomal vacuole. Furthermore, azurophil granules also contain defensins, which can permeabilize the bacterial membrane (14).

Neutrophils were originally only believed to be a part of the innate immune system, modulating host responses against pathogens in acute inflammation. However, in the last 15 years, it has been shown that neutrophils spontaneously or under appropriate stimulation can synthesize and release cytokines and chemokines, which are involved in directing incoming leukocytes and the regulation of the immune response $(15,16)$. Interleukin 12 (IL-12) is a key proinflammatory cytokine in host defense against mycobacterial infections, and it has been reported that neutrophils can release IL-12 (17). In the early stages, great numbers of neutrophils accumulate at the infection site, allowing these innate immune cells to contribute to large amounts of IL-12 $(7,18)$. The presence of IL-12 and interferon gamma (IFN- $\gamma$ ), another cytokine that is produced by natural killer cells, result in enhanced macrophage ability to kill bacteria, leading to the differentiation of helper $\mathrm{T}$ cells into $\mathrm{T}_{\mathrm{H}} 1$ cells (19). The development of a $\mathrm{T}_{\mathrm{H}} 1$ response is of great importance because it is associated with protective host immunity and the elimination of intracellular infectious agents $(18,20)$. Thus, neutrophil production and release of IL-12 during a bacterial infection is useful in the study of the organism's fate. 


\section{Mycobacterium smegmatis}

Mycobacterium smegmatis (M. smegmatis) is a rod-shaped, Gram-positive and acidfast saprophyte member of the rapidly growing division of the mycobacteria family. First isolated in 1884 by Lustgarten, M. smegmatis is in the non-obligate pathogens' sector of the environmental mycobacteria, and typically inhabit soils, natural waters, and municipal waters (21). Therefore, due to the ecological niches of $M$. smegmatis, humans are commonly exposed to it, even though this interaction rarely leads to illness or disease (22).

Nevertheless, despite typically being considered non-pathogenic, M. smegmatis retains the capacity to manipulate macrophage responses. It has the ability to survive and multiply within macrophages and delays the phagosomal acidification that accompanies the dumping of microbicidal agents $(23,24)$. Rare, but critical, cases of M. smegmatis-caused acute and chronic soft tissue, wound, or pleuropulmonary infections have been reported in both healthy and immunocompromised individuals (25).

Further emphasizing the presence of an established immune response against the frequently encountered M. smegmatis is the incidence of false positive Mantoux tuberculin tests. The skin test specifically assesses the adaptive immunity's T cell-mediated responses to the tuberculin protein, which is injected intradermally and is meant to detect previous exposure to Mycobacteria tuberculosis $(21,26)$. Interestingly, there is a high prevalence of false positive Mantoux tests due to individuals acquiring increased sensitivity and cross reactivity to the tuberculin protein from the recurrent exposure to environmental mycobacteria $(26,27)$. This reiterates the ability of environmental mycobacteria to modulate the immune system even though it is considered non-pathogenic.

\section{Phosphoinositol Lipoarabinomannan}

One of the primary ways immune cells interact with and detect invading bacteria is through the recognition of bacterial surface lipoglycans through pattern recognition receptors on immune cells. Recognition through specific receptors will activate different pathways and influence the outcome of not only the immune response but the fate of the bacteria as well. On the cell envelope, all mycobacterial species contain a variation of the glycolipid Lipoarabinomannan (LAM), which is typically associated with virulence. The cell envelope of M. smegmatis is studded with phosphoinositol-capped lipoarabinomannan 
(PILAM), which has been shown to play a role in modulating host macrophage responses during infection and resembles the structure of lipopolysaccharide (LPS) in Gram negative bacteria (28). Specifically in macrophages, PILAM is a potent inducer of IL-12 secretion, programmed cell death, and tumor necrosis factor alpha (TNF- $\alpha$ ), a cytokine that is released in the presence of bacteria and enhances phagocytosis $(17,29)$. Thus, observing the interactions between PILAM and human neutrophils could offer further, more specific insight as to how M. smegmatis may be involved in the innate system regulation.

Compared to other immune cells, neutrophils are a relatively understudied cell type, as is judged by the academic literature available. This is partly due to the inherent properties of human neutrophil research, such as their easy activation, short life span and inability for preservation. Furthermore, studying neutrophils require daily isolation from donor blood, which adds variability, because the neutrophil cell lines available lack some of the granule components that are present in donor-isolated cells. Furthermore, there is minimal information available regarding neutrophil interactions with environmental mycobacteria and the effect of the PI cap of LAM on neutrophils is unknown. M. smegmatis has been proposed as a model organism for the study of Mycobacteria tuberculosis, the causative agent of tuberculosis. M. smegmatis has a much shorter generation time, lower biosafety level requirements, many conserved virulence genes, and ease in genetic manipulation because of the shorter doubling time, making this avirulent microbe particularly appealing (30). The purpose of the present study was to make a comprehensive and comparison of the interaction between human primary neutrophils and viable M. smegmatis cells compared to stimulation with PILAM alone. 


\section{Materials \& methods}

- Buffers and Media:

- Krebs+ buffer: $\mathrm{NaCl}, \mathrm{KCl}$, Dextrose, NaPhosphate (Monobasic and dibasic) $\mathrm{CaCl}_{2}$ and $\mathrm{MgSO}_{4}$ dissolved in deionized water at $\mathrm{pH}=7.4$ and kept at $4{ }^{\circ} \mathrm{C}$ in depyrogenated bottles.

○ RPMI: RPMI-1640 (Life Technology), 0.1\% l-Glutamine, 10\% Heat inactivated Fetal Bovine Serum.

- MOPS + MgCl: (Molecular Sigma Biology, M8899) After dissolving MOPS powder, $1 \% \mathrm{MgCl}$ was added and the solution was adjusted to a $\mathrm{pH}$ of 7.2

- Incubations: Unless otherwise noted, all incubations were performed in a $37^{\circ} \mathrm{C}$ water bath with shaking.

\section{Neutrophil isolation}

Human neutrophils were isolated daily from healthy donors using Plasma-Percoll Gradients as previously described (31). Trypan blue exclusion indicated $>97 \%$ viability for the enriched cell populations, which contained $>95 \%$ neutrophils as judged by microscopic examination. Human donor recruitment, blood draws and the use of the material were performed according to guidelines approved by the Institutional Review Board of the University of Louisville. For all experimental conditions performed in this study, a concentration of neutrophils of $4 \times 10^{6}$ cells $/ \mathrm{mL}$ was used unless otherwise noted.

\section{Mycobacterium smegmatis}

- Bacteria Growth

Non-labeled and green fluorescent protein (GFP, from a plasmid)-expressing bacteria were grown to mid-log, exponential phase in 7H9 medium with 10\% ADC (Albumindextrose complex supplement, $10 \mathrm{~g}$ bovine serum albumin fraction $\mathrm{V}$ (BSA), $15 \mathrm{~g}$ D-glucose and $1.6 \mathrm{~g} \mathrm{NaCl}$ in $200 \mathrm{~mL}$ of water) at $37^{\circ} \mathrm{C}$ on a rotating drum. Before use, the bacteria were subjected to a light centrifugation ( $300 \mathrm{rpm}$ for $3 \mathrm{~min}$ ) and only the supernatant used to avoid any large clumps of bacterial cells. The total bacterial concentration was determined using a spectrophotometer where 0.D. $600=1$ is equivalent to $6 \times 10^{8}$ bacteria. All experiments with M. smegmatis were performed at a multiplicity of infection (MOI) of 6:1.

\section{- Bacteria Opsonization}

M. smegmatis was first centrifuged at $6000 \mathrm{~g}$ for $5 \mathrm{~min}$ at room temperature, the supernatant discarded and the bacteria pellet was resuspended in a 1:1 Krebs+ buffer and human serum (Complement technology, NHS) mixture. The cells were incubated without agitation for at least 20 minutes at room temperature, followed by pelleting and resuspension with Krebs+ two times. All bacterial conditions were performed with opsonized or non-opsonized M. smegmatis. 
- Heat-killed Bacteria

Opsonized M. smegmatis was placed in a $90^{\circ} \mathrm{C}$ water bath for 2 hours followed by a thorough mixing through a 21 gauge needle syringe to avoid any bacterial clumps. The bacteria suspension was separated into aliquots with a cell density of $2.4 \times 10^{8}$ cells $/ \mathrm{mL}$ and stored at $-80^{\circ} \mathrm{C}$.

\section{- UV-killed Bacteria}

Ten microliter drops of non-opsonized M. smegmatis solution $\left(10^{8}\right.$ cells $\left./ \mathrm{mL}\right)$ were pipetted in rows on a sterile petri dish. The petri dish was shielded with aluminum foil and placed six inches away from a UV lamp. The aluminum foil shield was removed so that each row was uncovered at specific times, and thus exposed each row to an increasing UV dosage. The drops of bacteria from each row were plated and their viability was assessed after 2 days by counting colony-forming units (CFU). It was determined that 3 min of UV exposure rendered the bacteria unviable. All subsequent experiments requiring UV-killed bacteria were completed with $M$. smegmatis exposed to UV light for $3 \mathrm{~min}$.

\section{Phosphoinositol Lipoarabinomannan (PILAM)}

PILAM was purchased from Invivogen (catalog \#tlrl-lams, version \#06C31-MT) in powder form. The PILAM powder was dissolved in sterile endotoxin-free deionized water to a concentration of $20 \mu \mathrm{g} / \mathrm{mL}$ and stored at $-20{ }^{\circ} \mathrm{C}$. For all experiments involving PILAM, concentrations of either $20 \mu \mathrm{g} / \mathrm{mL}$ or $40 \mu \mathrm{g} / \mathrm{mL}$ were used.

\section{Neutrophil degranulation/exocytosis}

- Measurement of azurophil granules, specific granules, and secretory vesicles Quantitative analysis of azurophil granule, specific granule, and secretory vesicle exocytosis was determined by measuring the increase in the presence of fluorescently tagged granule markers (CD63, CD66b, and CD35 respectively) in the cell plasma membrane using flow cytometry (FACS Calibur $\left.{ }^{\circledR}\right)$. Neutrophils were unstimulated, or stimulated with formyl-methionyl-leucyl-phenylalanine (fMLF (Sigma-Aldrich, $300 \mathrm{nM}$, for $5 \mathrm{~min}$ ) after the presence or absence of PILAM $(20 \mu \mathrm{g} / \mathrm{ml}$, or $40 \mu \mathrm{g} / \mathrm{ml})$ for 30 minutes, or in the presence or absence of opsonized or non-opsonized M. smegmatis for 15, 30, or 60 minutes. After stimulation, cells were incubated on ice in the dark for 45 minutes with fluorochrome-conjugated antibodies specific to each granule marker fluoresecein isothiocyanate (FITC)-conjugated anti-CD63 (azurophil granule, Ancell 215-040), FITCconjugated anti-CD66b (specific granule, Biolegend), and pycoerythrin (PE)-conjugated anti-CD35 (secretory vesicle, Biolegend 333406). Following antibody incubation, cells were washed with FTA buffer (BD 211248) + 0.1\% sodium azide (Sigma) and fixed with $1 \%$ paraformaldehyde (EMD PX0055-3) in FTA.

Formylated peptides, like fMLF, are derived from bacterial protein degradation and are a potent neutrophil chemoattractant and activator. Experimentally, fMLF alone was used as a positive control to measure neutrophil degranulation, as well as when combined with other stimuli to mimic the conditions of a multi-bacterial attack or chronic infection conditions. Only $20 \%$ of azurophil granules are mobilized upon neutrophil activation, and in vitro, it requires disruption of the actin cytoskeleton followed by fMLF stimulation. For 
the experimental conditions where azurophil granule exocytosis was measured, cells were pre-treated with the actin-disrupting drug, latrunculin A (1 $\mu \mathrm{M}, 30 \mathrm{~min}$, Sigma L5163).

- Measurement of degranulation of gelatinase granules using Enzyme-linked immunosorbent Assay (ELISA)

Degranulation of gelatinase granules was determined by measuring the release of gelatinase (matrix metalloprotease MMP-9) from unstimulated, or stimulated cells via enzyme-linked immunosorbent assays (ELISA, R\&D systems, DMP900). The same experimental conditions described above for the other granule subtypes were performed. Following stimulation cells were centrifuged at $6000 \mathrm{~g}$ for 30 seconds at room temperature, the supernatants were carefully removed from the cells into $100 \mu \mathrm{L}$ aliquot along with $1 \%$ phosphatase and 1\% protease inhibitors into sterile microcentrifuge tubes, and stored at $80^{\circ} \mathrm{C}$ until use.

\section{Respiratory burst}

- Phagocytosis-stimulated respiratory burst

For experiments using PILAM, neutrophils $\left(2 \times 10^{6}\right.$ cells $\left./ \mathrm{mL}\right)$ were unstimulated, or stimulated with opsonized Staphylococcus aureus (Pansorbin cells, Calbiochem, 507861, opsonized with plasma, MOI 6:1) for 10 minutes, in the presence or absence of PILAM (20 $\mu \mathrm{g} / \mathrm{ml}$, or $40 \mu \mathrm{g} / \mathrm{ml}, 30 \mathrm{~min}$ ), or in the presence or absence of either opsonized or nonopsonized M. smegmatis (MOI 6:1,10 min). Respiratory burst triggered by phagocytosis was detected by measuring ROS production with $50 \mu \mathrm{M} \mathrm{2} 2^{\prime}, 7^{\prime}$-dichlorofluorescein diacetate (DCF, Molecular Probes). DCF is a cell permeable non-fluorescent probe that becomes the fluorescent 2',7'-dichlorofluorescin upon oxidation by the respiratory burst in neutrophils. Phagocytosis-stimulated respiratory burst response was quantified by flow cytometry.

- ROS production in the phagosome

Neutrophils $\left(2 \times 10^{6}\right.$ cells $\left./ \mathrm{mL}\right)$ were attached to plasma-coated coverslips and challenged with GFP-tagged M. smegmatis (opsonized or non-opsonized, MOI 6:1) in RPMI media containing Nitro Blue tetrazolium (NBT, Sigma N6639) for one hour. Coverslips were fixed with methanol and then mounted onto a microscope slide and sealed with clear nail polish. NBT is a cytochemical stain that is reduced in the presence of oxidative species and will form deep blue deposits that outline intracytoplasmic deposits of ROS. Localization of the bacterium within neutrophil phagosomes was determined by confocal microscopy. Simultaneously, imaging of areas with NBT reduction was obtained using light microscopy. The confocal microscopy images distinguished if the areas of ROS production were the same as the location of $M$. smegmatis in phagosomes. Unstimulated neutrophils were utilized as negative control, and cells challenged with opsonized $S$. aureus or opsonized zymosan (Life Technologies), a particle that is usually found on the cell wall of yeast, were used as positive controls.

- Extracellular respiratory burst activity

A time course was performed to determine if viable $M$. smegmatis (opsonized, or non-opsonized, MOI 6:1) would induce superoxide release. For those experimental 
conditions, cells were unstimulated, or stimulated with fMFL (300 nM, $5 \mathrm{~min}$ ), or opsonized S. aureus (MOI 6:1), or opsonized or non-opsonized M. smegmatis (MOI 6:1), for 5, 15, or $30 \mathrm{~min}$. After each treatment, the cells were centrifuged and the supernatants collected, and the concentration of extracellular superoxide was calculated spectrophotometrically $(550 \mathrm{~nm})$ by measuring the color change that results from the reduction of Ferricytochrome C (FCC, $10 \mathrm{mg} / \mathrm{mL}$, Sigma C7752).

\section{Bacterial viability}

To determine if neutrophils were able to kill M. smegmatis, a bacterial viability kit (BacLight, Invitrogen Molecular probes L7012) was used taking advantage of the permeability properties of 2 nucleic acid dyes. One of the dyes, SYTO 9, is membrane permeable and will stain both live and dead bacteria cells. The other dye, propidium iodide (PI), is a non-permeable dye, which will only enter and stain bacterial cells that have compromised membranes and are classified as nonviable. When both dyes are combined, the PI will reduce the SYT09 and dead bacteria will stain red only, while viable bacteria will stain with a green color. This assay was modified specifically for M. smegmatis from Johnson and Criss (32).

Neutrophils ( $2 \times 10^{6}$ cells $\left./ \mathrm{mL}\right)$ were attached to plasma-coated coverslips and challenged with opsonized $M$. smegmatis for 30 minutes in 24-well plates. The cells were treated with the combination of dyes, $30 \mu \mathrm{M}$ PI and $2 \mu \mathrm{M}$ SYTO 9 mixed into MOPS buffer with $0.1 \%$ saponin from quillaja bark (Sigma Life science S4521-25G). Following the incubation time, the cells were stained in the dark for 15 minute at room temperature. Two washes were performed with MOPS buffer to remove excess dye staining. The coverslips were removed from the plate wells, placed on microscope slides, sealed with clear nail polish and analyzed with confocal microscopy. The controls for the assay were live and heat-killed bacteria alone.

\section{IL-12 Cytokine release}

Supernatants and cell lysates were collected from neutrophils that were unstimulated, or stimulated with interferon gamma (IFN- $\gamma, 50 \mathrm{ng} / \mathrm{mL}$ (BD Pharmingen) and LPS $(1 \mu \mathrm{g} / \mathrm{mL})$ in the presence or absence of LAM $(20 \mu \mathrm{g} / \mathrm{mL})$ or M. smegmatis (opsonized or non-opsonized) in RPMI media overnight. IFN- $\gamma$ and LPS stimulation was used as a positive control that will trigger the release of IL-12 by human neutrophils.

All experimental manipulations were performed using sterile technique in a tissue culture hood. After 24 hours of stimulation, cells were centrifuged, their supernatant removed and aliquoted with 1\% protease and phosphatase inhibitors each. The cell pellet was further processed by disrupting the cells at $4{ }^{\circ} \mathrm{C}$ for 30 minutes on a rotator with lysis buffer (AKT buffer $+1 \%$ protease inhibitor, $1 \%$ phosphatase inhibitor, $1 \% \mathrm{Na}_{3} \mathrm{VO}_{4}, 1 \%$ AEBsf). Subsequently, the lysed cells were centrifuged and the lysate supernatant collected and stored at $-80^{\circ} \mathrm{C}$. IL-12 levels were measured using an ELISA kit (BioLegend 431704).

\section{Statistical Analysis}

Statistical differences among experimental conditions, and/or time points, were analyzed by ANOVA and the Tukey multiple-comparison (GraphPad Software, San Diego, CA). Differences were considered significant at the level $\mathrm{P}<0.05$. 


\section{Results and Discussion}

The immune system is very effective at eliminating $M$. smegmatis, which is evidenced by the rarity of $M$. smegmatis-derived infections. However, the exact mechanism for this killing is unknown, and even less is known about the mechanism of pathogenesis during those rare cases where M. smegmatis is able to cause acute and chronic infections. As the first responders of innate immunity, neutrophils are a key player in the clearing of microbe intruders, making them relevant to studies between the human immune system and environmental mycobacteria.

\section{DEGRANULATION/EXOCYTOSIS}

Following stimulation by M. smegmatis or PILAM, the degranulation of the four granule subtypes was analyzed either by using flow cytometry or ELISA.

\section{Neither PILAM nor M. smegmatis have an effect on secretory vesicle exocytosis}

A.

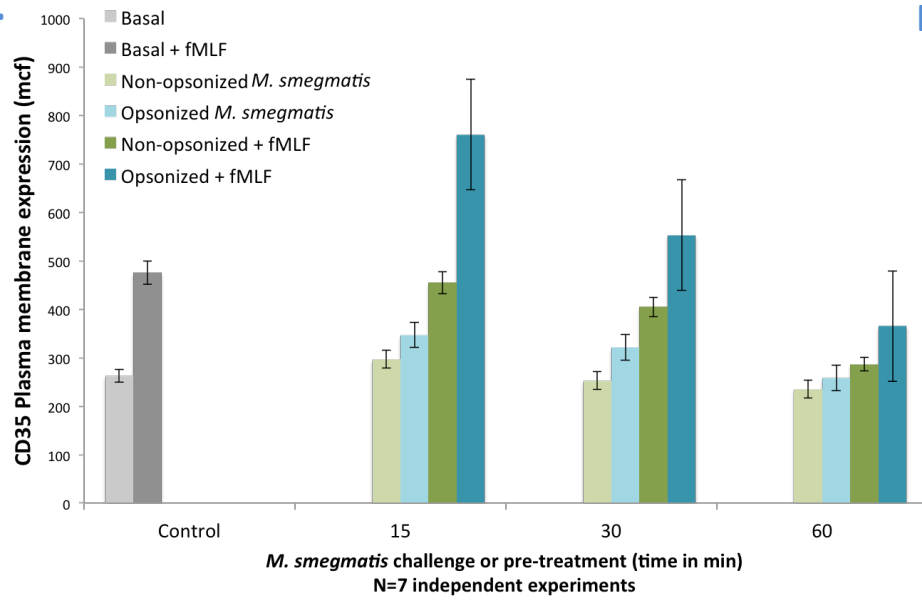

B.

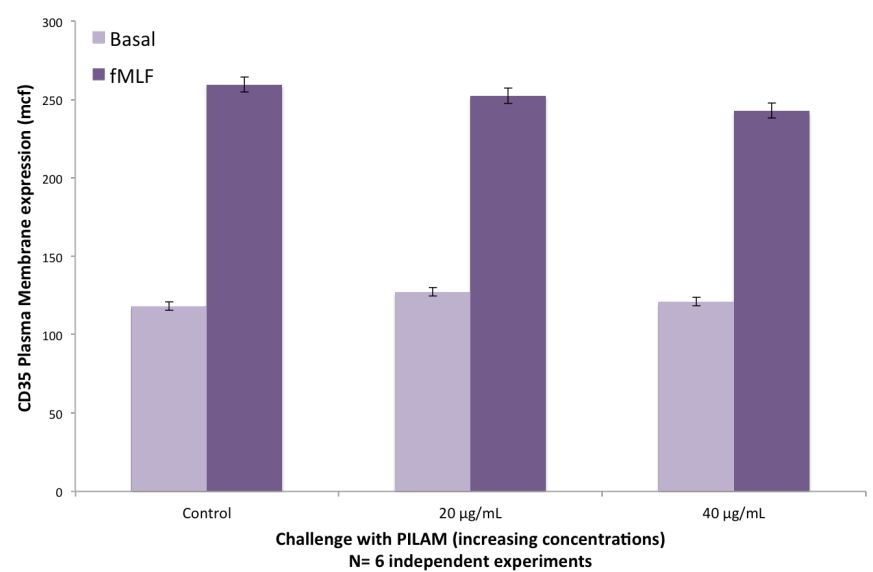

Fig. 1: Exocytosis of Secretory vesicles. Human neutrophils ( $4 \times 10^{6}$ cells $\left./ \mathrm{ml}\right)$ were unstimulated (Basal), or stimulated with formyl-methionyl-leucylphenylalanine (fMLF, $300 \mathrm{nM}, 5 \mathrm{~min}$ ), in the presence or absence of opsonized or non-opsonized M. smegmatis at an MOI of 6:1 (A) or presence or absence of increasing concentrations of PILAM (B). Exocytosis of secretory vesicles (CD35 plasma membrane expression) was measured by flow cytometry. Data are expressed as mean \pm SEM of the mean channel of fluorescence ( $\mathrm{mcf}$ ).

Embedded on the secretory vesicles are many receptors that are incorporated into the cell's membrane after degranulation. These receptors transform naïve neutrophils into extremely sensitive cells, primed for detecting any microbe or signal from the environment with greater responsiveness. However, after exposure to only opsonized or non-opsonized $M$. smegmatis, neutrophils had similar levels of the CD35 marker, which is characteristic of the secretory vesicles, when compared to the untreated neutrophils (Fig. 1A). This indicates that M. smegmatis presence is not a source of neutrophil membrane sensitization. Furthermore, it suggests that the mechanism of elimination for M. smegmatis 
does not require the recognition of the bacterium by the receptors that are contained in the secretory vesicles.

Incubation for 15 min with opsonized M. smegmatis followed by subsequent 5 min fMLF stimulation resulted in an enhanced exocytosis of secretory vesicles compared to fMLF alone (Fig. 1 A). These results indicate that opsonized $M$. smegmatis was able to prime the neutrophil response. As time progressed, there is a decreasing trend in the exocytosis of these granules, but this could be due to the induction of endocytosis rather than inhibition of exocytosis. If bacteria are engulfed, there will be a decrease in the receptors available for fMLF stimulation, thus explaining the decrease in plasma membrane expression.

Similarly to the response seen with the whole bacterium, the presence of increasing concentrations of PILAM is unable to effect a change in secretory vesicle exocytosis compared to untreated cells or fMLF-treated cells (Fig. 1B). Since the in vitro fMLF-stimulation of neutrophils mimics the activated cell phenotype induced by bacteria, the stable secretory vesicle release suggests that the presence of PILAM alone will neither enhance nor dampen the reactivity of neutrophils against $M$. smegmatis.

Non-opsonized M. smegmatis significantly induced gelatinase granule release.
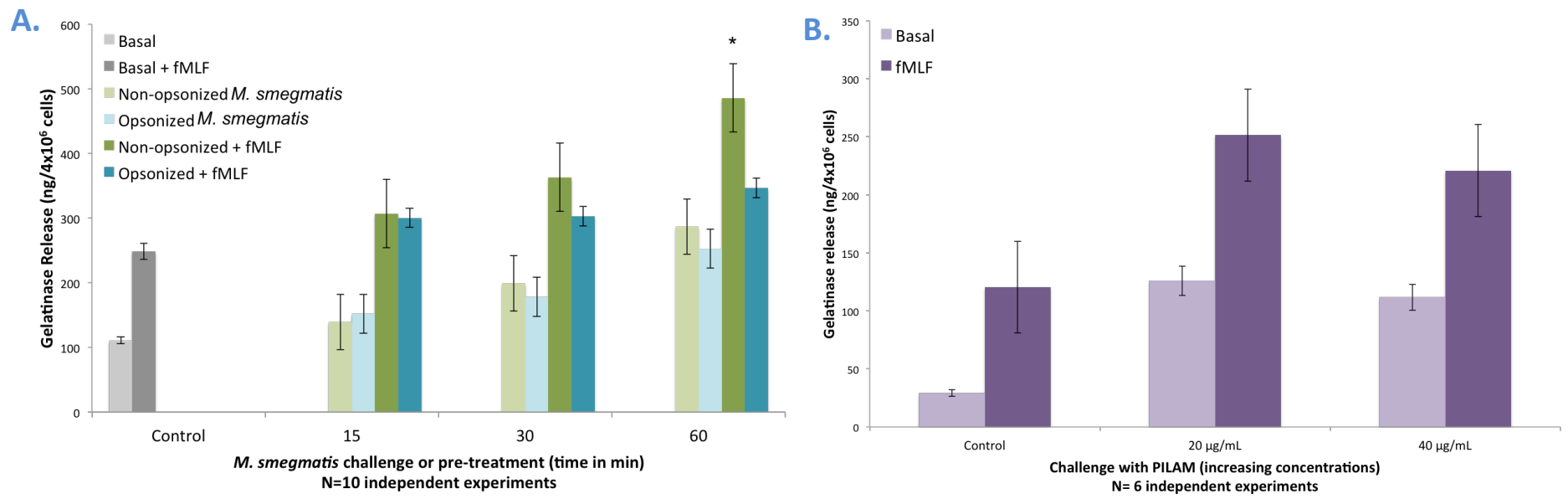

Fig. 2: Exocytosis of Gelatinase granules. Human neutrophils ( $4 \times 10^{6}$ cells $\left./ \mathrm{ml}\right)$ were unstimulated (Basal), or stimulated with formyl-methionylleucyl-phenylalanine (fMLF, $300 \mathrm{nM}, 5 \mathrm{~min}$ ), in the presence or absence of opsonized or non-opsonized M. smegmatis at an MOI of 6:1 (A) or presence or absence of increasing concentrations of PILAM (B). Exocytosis of gelatinase granules (MMP-9 release) was measured by ELISA. Data are expressed as the mean \pm SEM of $\mathrm{ng} / \mathrm{ml}$ of gelatinase release; ${ }^{*} \mathrm{p}<0.05$ compared to control.

Gelatinase granules are often exocytosed by neutrophils for tissue migration after transendothelial movement because the enzymes within these granulesprotease. Our data showed that 60 min of bacterial challenge (opsonized or non-opsonized) resulted in a similar release of MMP-9 as fMLF-stimulation, which was used as a positive control for this assay (Fig. 2A). In addition, a significant increase of MMP-9 release was observed when neutrophils were pre-treated with non-opsonized 
bacteria for $60 \mathrm{~min}$ followed by fMLF-stimulation (Fig. 2A). Similar results were observed when neutrophils were challenged with just the glycolipid PILAM (Fig. 2B). PILAM alone was able to induce gelatinase granule exocytosis and was able to enhance the MMP-9 release after fMLF-stimulation (Fig. 2B). This finding describes a defensive host mechanism because the additional gelatinase release makes movements throughout the tissue easier and thus the infection can be cleared much faster. However, significant exocytosis of these granules has been linked with host tissue damage, and gives some insight into the role that $M$. smegmatis may play in its rare chronic infections. The presence of this environmental bacterium induces neutrophils to secrete extracellular matrix-degrading proteins that could worsen a developing or established infection and prevent resolution.

Furthermore, the difference seen between the opsonized or non-opsonized bacterium could be explained by the fact that opsonization aids in recognition and phagocytosis, meaning that neutrophils will not have to travel as much within tissue to find and engulf the intruders. If the bacteria are nonopsonized, neutrophils could have greater difficulties locating and internalizing these microbes, meaning that the signals of bacterial presence would continue to induce inflammation, further stressing the neutrophils. In addition, some of the proteases that are released from gelatinase granules are involved in shedding of IL-8, L-selectin, and CD35. This cell surface reorganization may further activate other receptors. Together, the combined release of the gelatinase would result in detrimental host tissue damage.

\section{Neither M. smegmatis nor PILAM induced specific granule exocytosis}
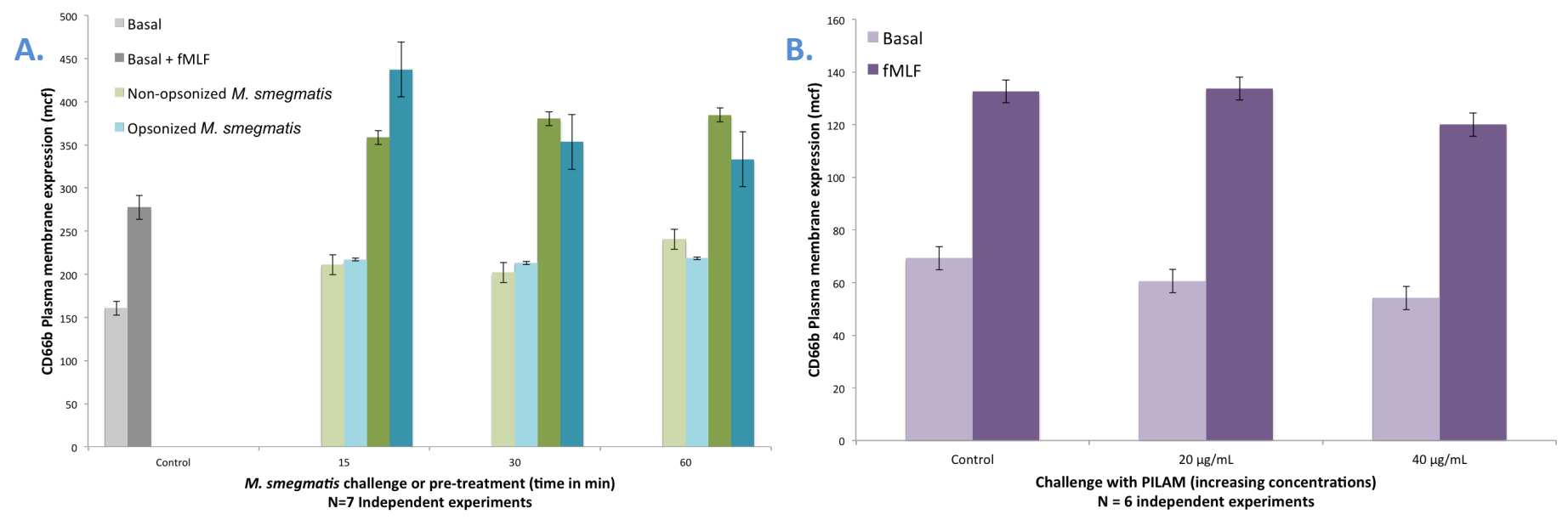

Fig. 3: Exocytosis of Specific Granules. Human neutrophils ( $4 \times 10^{6}$ cells/ml) were unstimulated (Basal), or stimulated with formyl-methionyl-leucylphenylalanine (fMLF, $300 \mathrm{nM}, 5 \mathrm{~min}$ ), in the presence or absence of opsonized or non-opsonized M. smegmatis at an MOI of 6:1 (A) or presence or absence of increasing concentrations of PILAM (B). Exocytosis of specific granules (CD66b plasma membrane expression) was measured by flow cytometry. Data are expressed as mean \pm SEM of the mean channel of fluorescence (mcf).

Unlike the secretory vesicles and the gelatinase granules, specific granules contain high concentration of microbicidal proteins. Characterized by the presence of lactoferrin and other 
bacteriostatic or bactericidal compounds, the mobilization of this granule is highly controlled. Therefore, it was not surprising that neither opsonized bacteria, non-opsonized bacteria, nor PILAM were able to induce the extracellular release of these granule components compared to untreated cells (Fig. 3A and 3B). Moreover, exposure of neutrophils to PILAM or M. smegmatis did not further incite the expression of CD66b on neutrophil membranes in fMLF-stimulated cells (Fig. 3A and 3B). This suggests that neither the whole organism nor PILAM specifically, is able to give sufficient signaling for lactoferrin release into the extracellular matrix when acting alone or as a cohort with another microbe. The mobilization of these granules may be reserved for much more pathogenic organisms due to the potential host tissue destruction from exocytosis. Interestingly, this finding confirms previous data showing that during neutrophil exposure to M. smegmatis at an MOI of 50, specific granules exocytosis is not enhanced by $M$. smegmatis whether it is opsonized or non-opsonized (8). The current experiments were performed at an MOI of 6, suggesting that neutrophil specific granule responses remain unchanged even when they face a steep increase in microbes to combat.

\section{Non-opsonized and UV-killed $M$. smegmatis significantly induced fMLF-stimulated azurophil granule} exocytosis in a time dependent manner whereas PILAM inhibited fMLF-stimulated degranulation.
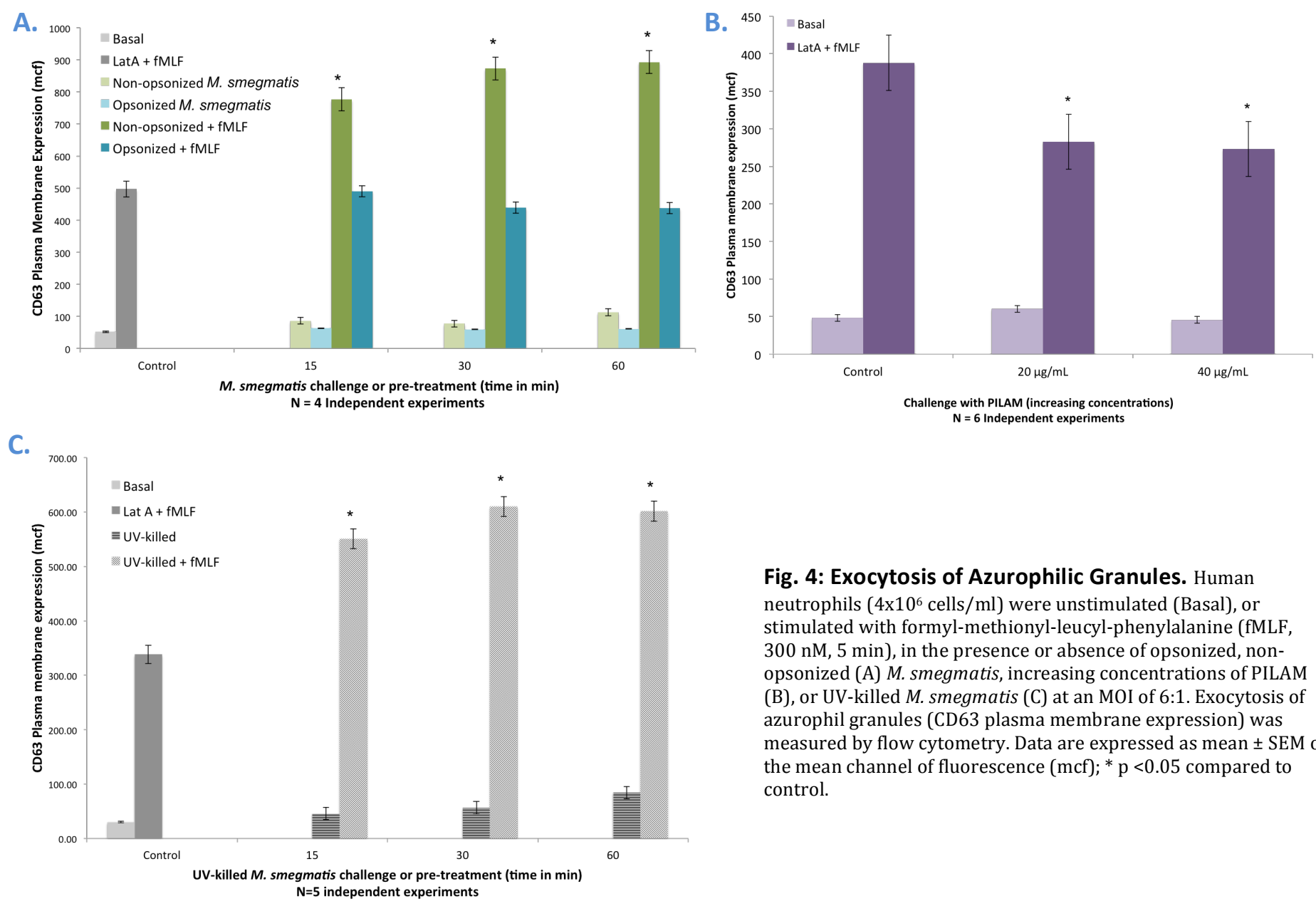

Fig. 4: Exocytosis of Azurophilic Granules. Human neutrophils $\left(4 \times 10^{6}\right.$ cells $\left./ \mathrm{ml}\right)$ were unstimulated (Basal), or stimulated with formyl-methionyl-leucyl-phenylalanine (fMLF, $300 \mathrm{nM}, 5 \mathrm{~min}$ ), in the presence or absence of opsonized, nonopsonized (A) M. smegmatis, increasing concentrations of PILAM (B), or UV-killed M. smegmatis (C) at an MOI of 6:1. Exocytosis of azurophil granules (CD63 plasma membrane expression) was measured by flow cytometry. Data are expressed as mean \pm SEM of the mean channel of fluorescence (mcf); ${ }^{*} \mathrm{p}<0.05$ compared to control. 
The deadliest granule contents, myeloperoxidase and defensins, are within the azurophil granules. Due to their effectiveness as sterilants, the release of this granule's content into the extracellular matrix is the most challenging. Nevertheless, despite an inability to mobilize the granules independently as previously seen in other studies (8), pre-incubation of neutrophils with non-opsonized M. smegmatis was able to increase the fMLF-stimulated plasma membrane expression of CD63 (Fig 4A). This finding is particularly supportive of the role of $M$. smegmatis in chronic illnesses because the increased release of these granules in an ongoing infection would be a major source of host tissue damage and inflammation. The data also shows that from 15 to 60 minutes post-infection, the amount of azurophil exocytosis does not increase, suggesting that the primary granule response will reach its maximum exocytosis almost immediately after encountering $M$. smegmatis. This robust unloading of myeloperoxidase shortly after the initial encounter offers a mechanism for the cases of acute inflammation with $M$. smegmatis. Because the added presence of $M$. smegmatis induces the amplified azurophil degranulation, it could also inadvertently be acting as a catalyst to spur neutrophils to eliminate the bacteria and clear the infection sooner than if M. smegmatis would not have been present.

UV-exposure affects the mycobacterial DNA, making it unviable, and thus preventing the bacteria from creating and secreting any compounds in a stress-state after interacting with neutrophils. When the outer membrane components remain intact, experiments with the UV-killed organism give insight into the relevant question of whether or not M. smegmatis actively regulates neutrophil's azurophil exocytosis. Similar to the viable M. smegmatis, testing with the UV-killed microbe also displayed an augmented azurophil degranulation in FMLF-activated neutrophils (Fig. 4C). This outcome is significant because it indicates that the viable bacterium does not actively control this reaction and that signaling pathways triggered by receptor recognition or phagocytosis are involved in the regulation of degranulation.

Since a membrane component is responsible for the observed effect on primary granule exocytosis, treatment of neutrophils with PILAM was a critical facet of this work. Pre-treatment of neutrophils with PILAM decreased CD63 plasma membrane expression in fMLF-treated neutrophils, but PILAM treatment alone had no effect (Fig. 4B). Although the decrease on CD63 plasma membrane is less pronounced and did not reach statistical significance, similar results were observed when neutrophils were pre-incubated for 60 min with opsonized M. smegmatis (Fig. 4A). In other, more pathogenic mycobacterial species, the LAM glycolipid is associated with virulence, so in the context of the mycobacterial genus, it becomes relevant that PILAM decreased the azurophil degranulation because it could provide the bacterium with a mechanism for evading neutrophil killing. In addition, the difference 
observed in the regulation of granule exocytosis between challenging neutrophils with opsonized vs nonopsonized bacteria emphasizes the importance of the opsonization process in determining the fate of the bacterium and the degree of neutrophil activation. Additionally, in the rare cases of infection with $M$. smegmatis, the decreased azurophil granule exocytosis would also protect the other bacteria in the environment, prolonging the infection. When comparing the result of treatment with PILAM alone and with UV-killed M. smegmatis, it becomes clear that other components of the membrane could have a greater effect on the fate of $M$. smegmatis since the opposite effects on exocytosis were observed.

\section{Respiratory Burst Response}

The most potent microbicidal mechanisms employed by neutrophils is the intracellular and extracellular release of ROS. This is induced by activation of the respiratory burst against M. smegmatis, and is a critical component in the study of the cell-bacteria interaction. Due to the rarity of M. smegmatis infection, an increased ROS production intracellularly or extracellularly was expected. For comparison, the experiments were performed alongside opsonized and non-opsonized S. aureus, another Grampositive microbe that is known to induce a robust oxidative burst in neutrophils.

Opsonized M. smegmatis and PILAM significantly induce a time-dependent increase in intracellular oxidants production
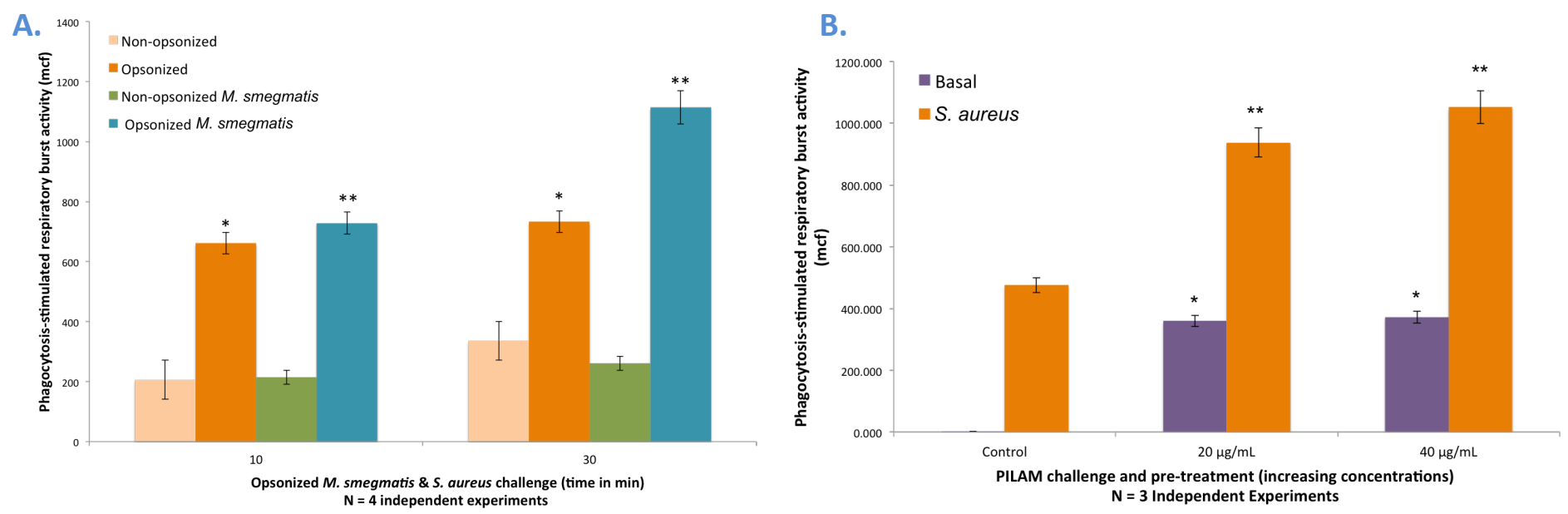

Fig. 5. Intracellular oxidants production. Human neutrophils $\left(2 \times 10^{6} \mathrm{cells} / \mathrm{ml}\right)$ were exposed to 2'-7'-dichlorofluorescin diacetate (DCF) in the presence of human serum opsonized or non-opsonized M. smegmatis or heat-killed, opsonized $S$. aureus at an MOI of 6:1 (A) or increasing concentrations of PILAM $(20 \mu \mathrm{g} / \mathrm{mL}$ or $40 \mu \mathrm{g} / \mathrm{mL})$ alone or followed by stimulation with $S$. aureus (B). Intracellular oxidants production was determined by flow cytometry measuring the oxidation of DCF. Data are expressed as mean \pm SEM of the mean channel of fluorescence (mcf); ${ }^{*} \mathrm{p}<0.05$ compared to non-opsonized S. aureus, ${ }^{* *} \mathrm{p}<0.05$ compared to non-opsonized M. smegmatis (A). After exposure to PILAM (B), ${ }^{*} \mathrm{P}<0.05$ compared to basal control, ${ }^{* *} \mathrm{P}<0.05$ compared to $S$. aureus-stimulated control. Data expressed as mean \pm SEM of the mean channel fluorescence 
Both non-opsonized Gram-positive bacteria induced a moderate intracellular oxidative response, with minimal increase in ROS production between the 10 and 30-minute time points (Fig. 5A). Regardless of the time point, however, the opsonization of either bacterium substantially increased the ROS response when compared to their non-opsonized counterparts at the same time point. This emphasizes the significance that the type of microbial recognition defines the fate of the microbe because complement recognition by the complement receptor led to a phagocytic event that culminated in greater ROS production than the internalization of non-opsonic bacteria. Moreover, after 30 min challenge with opsonized M. smegmatis, an even greater oxidative response was seen when juxtaposed against opsonized $M$. smegmatis after 10 minutes and opsonized $S$. aureus after 30 minutes. Therefore, in a time dependent manner, opsonization of $M$. smegmatis significantly increased neutrophils' intracellular oxidant production when compared to non-opsonized M. smegmatis and opsonized S. aureus at 30 minutes. Hence, these results indicate a greater efficiency of reactive oxidative species production when the bacteria have been recognized and internalized with the help of complement.

PILAM also displayed some ability to induce intracellular oxidant production (Fig. 5B). Increasing PILAM concentrations significantly increased ROS response independently, which suggests that PILAM is a potent inducer and a crucial factor in neutrophil oxidative burst activity against $M$. smegmatis. This finding is even more noteworthy because previous studies have shown that mannose-capped LAM (ManLAM) and arabinose-capped LAM (AraLAM) from other mycobacterial species do not significantly activate the NADPH-oxidase activity as measured by superoxide production (28). This difference could be explained by different binding to the neutrophil receptor via the caps attached to the LAM structure. The ability of PILAM to activate the NADPH response may provide one of the factors that make M. smegmatis more susceptible to neutrophils when compared to its mycobacterial counterparts. Conflicting with this finding, there is some evidence to suggest that human neutrophils kill invading mycobacteria by oxygenindependent methods only (33). A study from the University of Illinois at Chicago showed that neutrophils from chronic granulomatous disease (CGD), which are unable to produce the oxygen radicals of the respiratory burst, were equally efficient at killing mycobacteria as normal neutrophils (34).

Additionally, PILAM pre-treatment before challenge with opsonized S. aureus significantly increased neutrophil ROS production (Fig. 5B). The expanded activation of intracellular ROS from PILAM pre-treated cells demonstrates that the mycobacterial glycolipid has a priming effect on neutrophils unlike its LAM relatives, ManLAM and AraLAM (28). While in the primed state, there is no increase in the NADPH oxidase activity initially; however, primed neutrophils' subsequent microbial interactions provoke an amplified response when compared to non-primed, activated cells. Previous work determined that LPS-primed neutrophils were able to kill 80\% of M. smegmatis after 30 min (33). 
Intracellular oxidants accumulate within phagosomes that contain $M$. smegmatis

\section{A. Untreated}

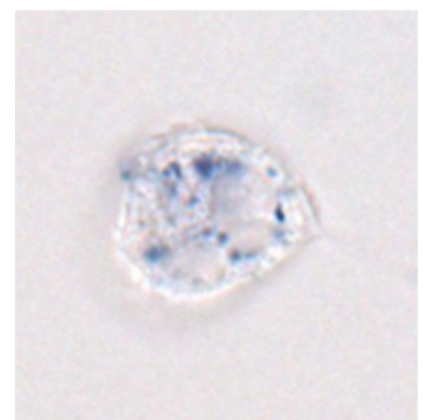

C. Heat-killed, opsonized S. aureus
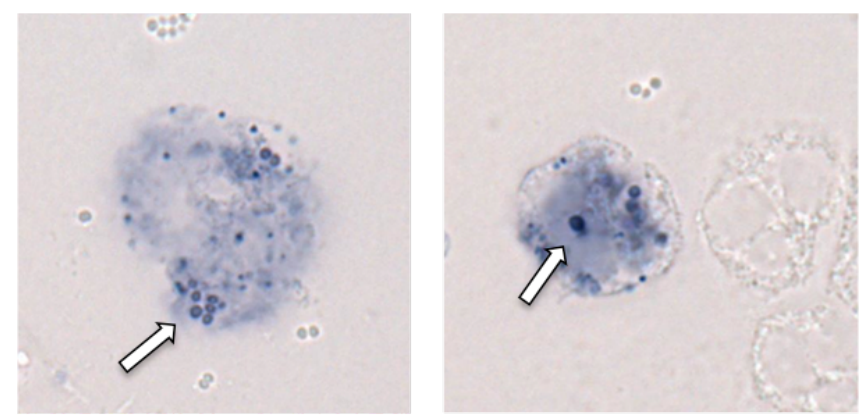

D. Non-opsonized M. smegmatis
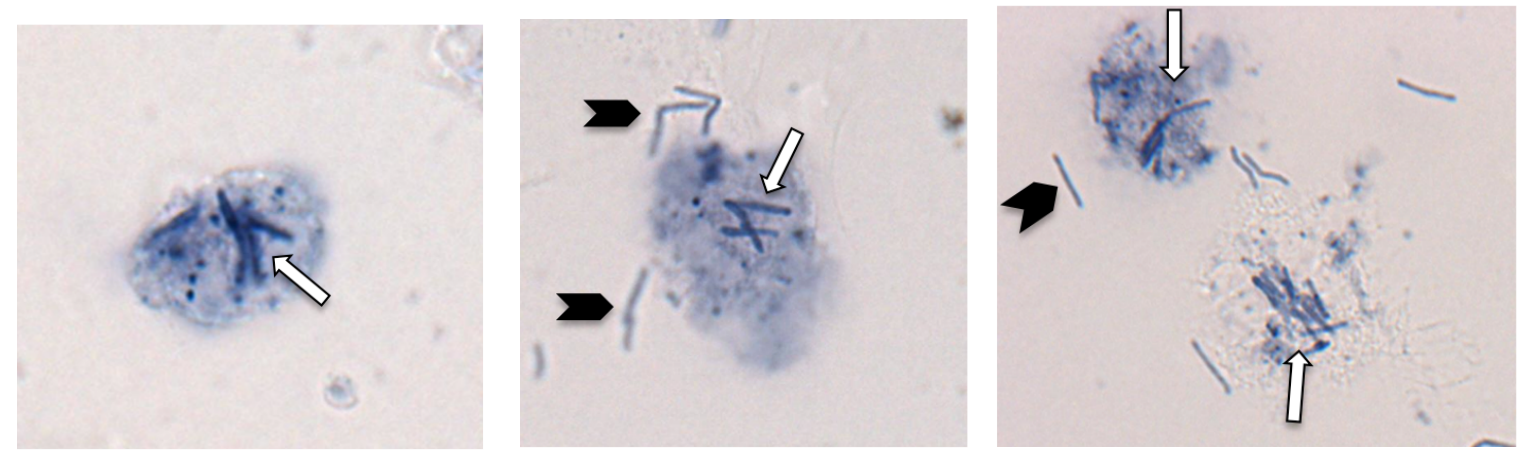

E. Opsonized M. smegmatis
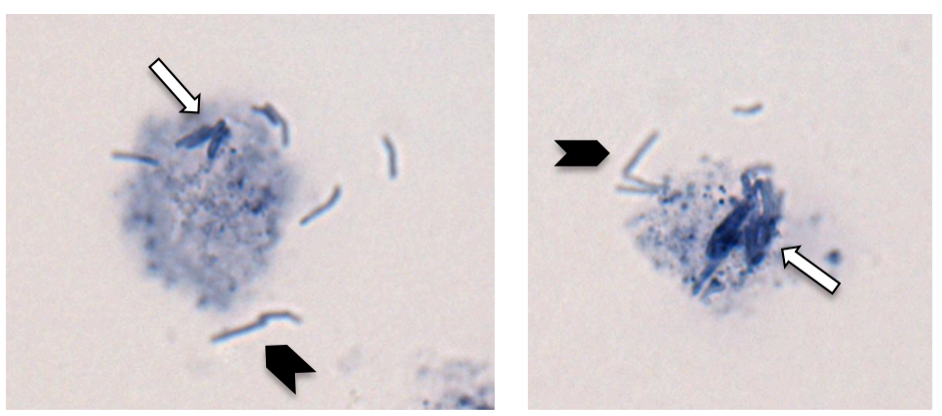

\section{B. Opsonized Zymosan}

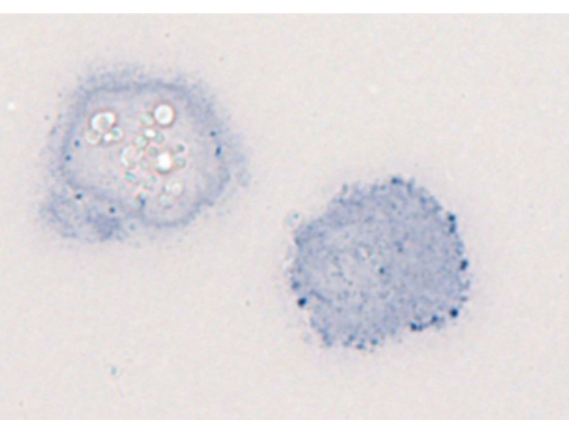

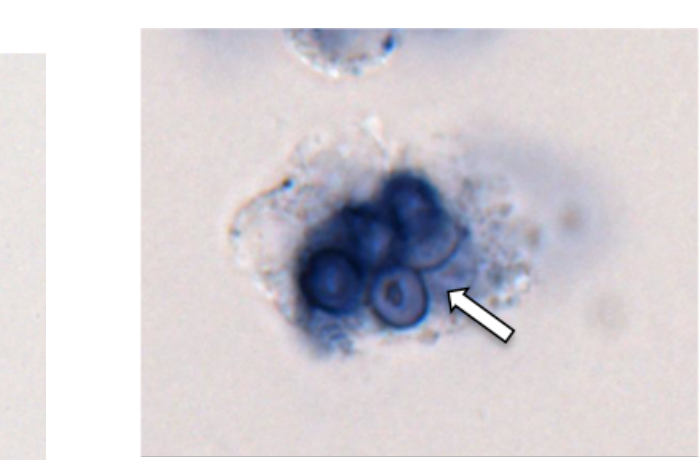

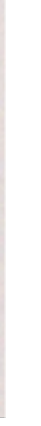

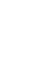


Another mycobacterial species, the highly pathogenic Mycobacteria tuberculosis, is known to inhibit phagosome-lysosome fusion through its LAM protein, ManLAM, in macrophages by inhibiting the recruitment of lysosomal components (35). The increase in neutrophil's intracellular ROS by opsonized M. smegmatis and PILAM confirmed that intracellular oxidant production might be involved in the degradation of the bacterium. To confirm the suspected ROS formation at the phagosome; neutrophils were visualized with light microscopy post-challenge with M. smegmatis in the presence of nitro-blue tetrazolium (NBT). The formation of navy blue deposits inside M. smegmatis-containing phagosomes would indicate oxidants were produced inside those phagosomal structures. The successful maturation of the phagosome would offer a possible mechanism for killing M. smegmatis that differs from what has been seen in macrophages when they are exposed to M. tuberculosis or ManLAM.

Untreated cells appeared mostly clear or a very light shade of blue, with very small and scarce dark blue deposits (Fig. 6 A) whereas the positive controls, opsonized S. aureus and opsonized zymosan, clearly exhibited small circular and large oval navy blue deposits on the phagosomal structures respectively (Fig. 6 B,C). Even with uptake of either compound into the phagosome, the rest of the cell retained a clear or light blue coloring indicative that the oxidation event was only taking place at the compartment containing the infectious particle. When neutrophils were challenged with M. smegmatis, the cells displayed rod-shaped, dark blue NBT deposits within the cytoplasm that are indicative of ROS production at a phagosome; however, when $M$. smegmatis was found outside of neutrophils, it also stained dark blue (Fig. 6 D, E). The NBT staining of extracellular M. smegmatis raised doubts concerning the intracellular respiratory burst at the phagosome.

Wild type M. smegmatis was substituted with GFP-tagged M. smegmatis, and confocal microscopy was used to acquire merged images that eliminated any doubt that there was co-localization of the fluorescently green bacteria and the dark blue deposits in the bright field microscopy channel (Fig. 5). Thus, the accumulation of intracellular oxidants at the phagosome was visually confirmed. These findings not only further supporting the hypothesis that activation of the respiratory burst response is one of the mechanisms used to eradicate M. smegmatis, but they also made a clear distinction that PILAM does not behave in neutrophils like the virulent ManLAM does in macrophages. By not inhibiting the phagosome maturation process, $M$. smegmatis is more susceptible to elimination and probably one of the reasons it is considered less pathogenic than other mycobacteria. 


\section{A. Untreated}

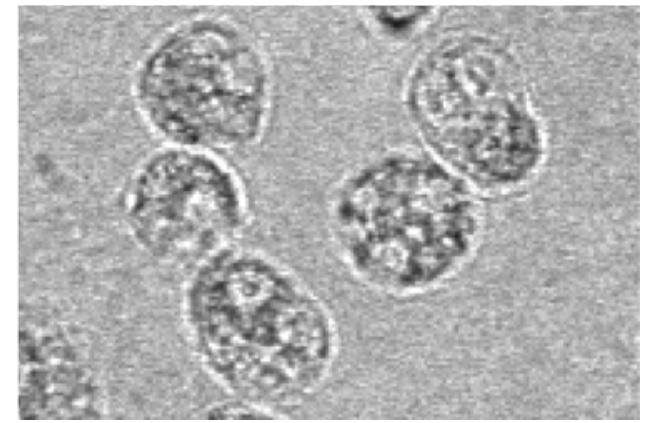

$\Longrightarrow$ NBT positive phagosomes

Extracellular M. smegmatis

\section{B. Non-opsonized M. smegmatis}
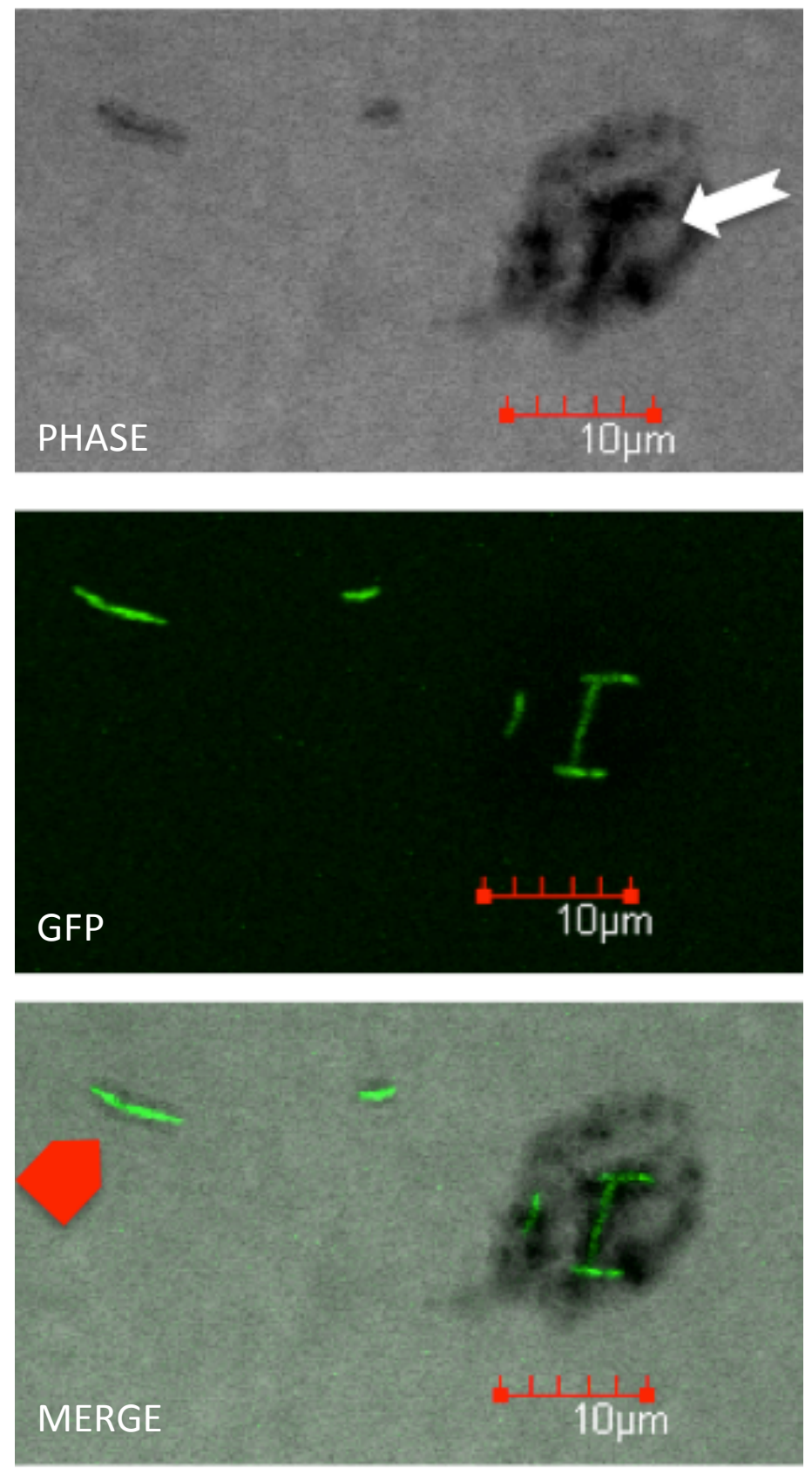

\section{Opsonized M. smegmatis}
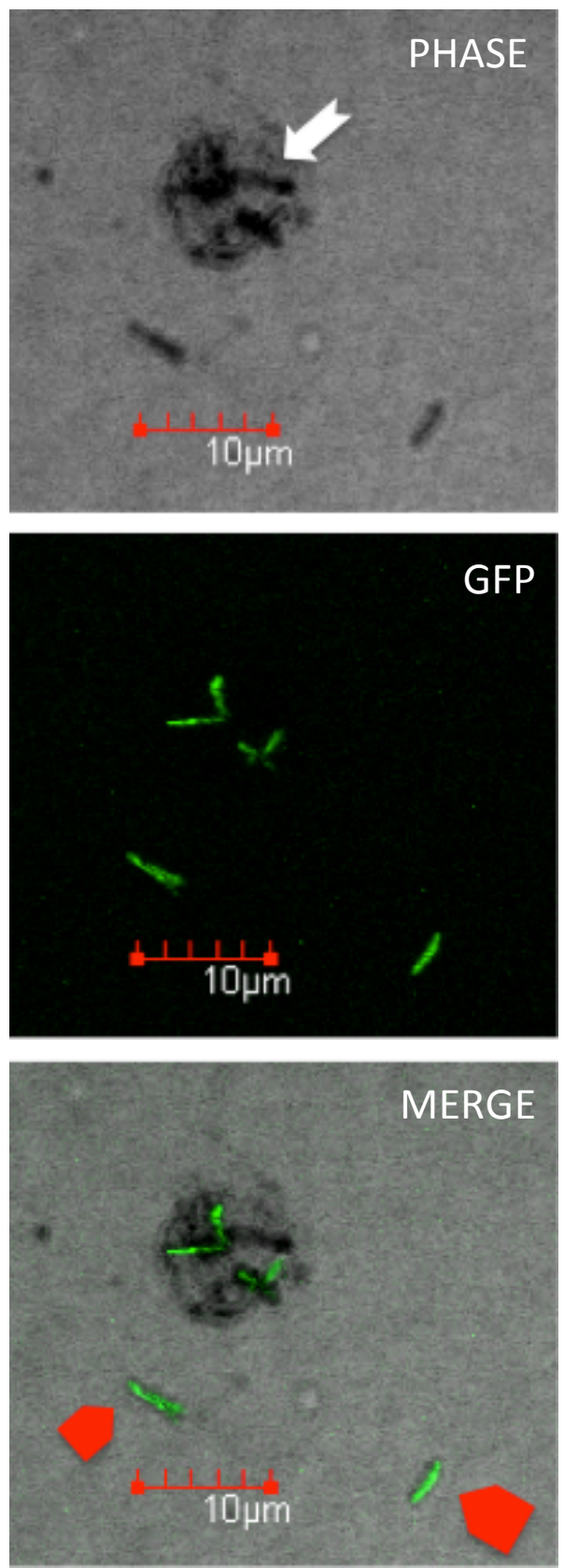

Fig.7 NBT staining of opsonized \& non-opsonized GFP-tagged $\boldsymbol{M}$. smegmatis. Neutrophils $\left(2 \times 10^{6} \mathrm{cell} / \mathrm{ml}\right)$ were unstimulated (UT)(A), or stimulated with non-opsonized M. smegmatis (MOI 6:1) (B), or serum opsonized M. smegmatis (MOI 6:1)(C) for 60 min in NBT media. White arrows show NBT-positive phagosomes; red arrows show M. smegmatis. 


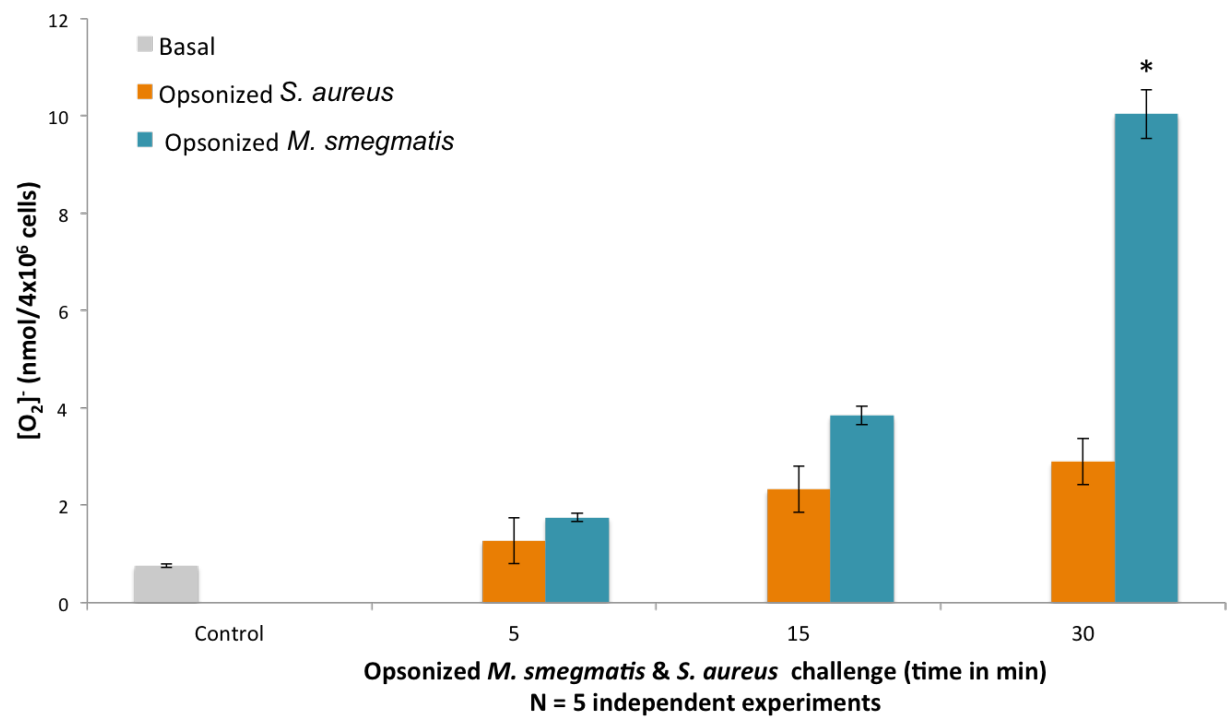

Fig. 8 Extracellular oxidants production. Human neutrophils ( $4 \times 10^{6}$ cells $\left./ \mathrm{ml}\right)$ were exposed to or ferricytochrome $c$, in the presence of human serum opsonized M. smegmatis or $S$. aureus at an MOI of 6:1. Superoxide release was measured as the superoxide dismutaseinhibitable reduction of ferricytochrome c. Data are expressed as mean \pm SEM in $\mathrm{nmol} / 4 \times 10^{6}$ cells of superoxide release, ${ }^{*} \mathrm{P}<0.05$ compared to all the experimental conditions.

Alternately, activation of the NADPH oxidase at the plasma membrane results in the release of newly formed ROS to the extracellular space rather than into the phagosome. Similar to the results obtained from the intracellular oxidative burst response, opsonized $M$. smegmatis induced a timedependent, increasingly robust extracellular oxidant release when compared to opsonized $M$. smegmatis at 5 and 15 minutes, and opsonized S. aureus at all time points (Fig. 8). The superoxide ions produced react quickly with other molecules, making this stimulant-dependent process effective at killing extracellular microbes but also harmful to host tissues if left unchecked. In fact, although this response typically leads to the elimination of M. smegmatis, this extremely potent extracellular respiratory burst caused by $M$. smegmatis could further explain its role in the rare acute and chronic inflammation and tissue damage in soft tissue, wounds and the pleuropulmonary area.

\section{CYTOKINE RELEASE}




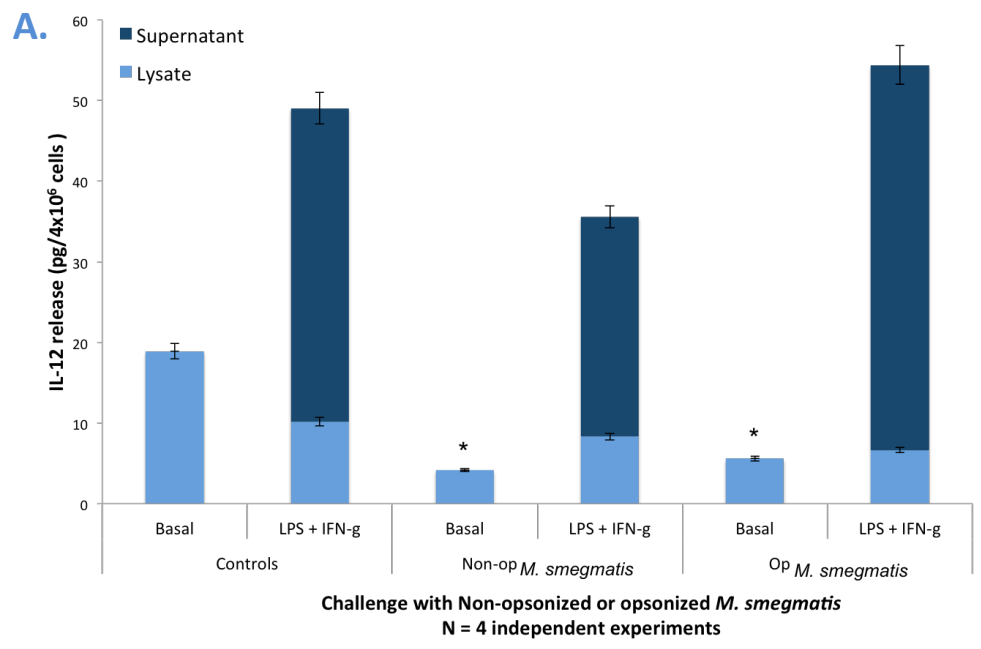

B.

Fig.9 IL-12 production and release. Human neutrophils $\left(4 \times 10^{6}\right.$ cells $\left./ \mathrm{ml}\right)$ were unstimulated (Basal), or stimulated with Lipopolysaccharide (LPS, $1 \mu \mathrm{g} / \mathrm{ml}$ ) + Interferon- $\gamma($ IFN-g, $50 \mathrm{ng} / \mathrm{ml}$ ), or pre-treated with non-opsonized (Non-op $M$. smegmatis), serum opsonized (Op) M. smegmatis (MOI 6:1, $30 \mathrm{~min}$ )(A) or $20 \mu \mathrm{g} / \mathrm{mL}$ of PILAM followed by LPS + IFN-g stimulation (B). Supernatants and cell lysates were collected after $24 \mathrm{~h}$ stimulation and IL-12 levels measured by ELISA. Data are expressed as mean \pm SEM of IL-12 levels (pg/ml), ${ }^{*} \mathrm{p}<0.05$ compared to lysate of non-op and op-M. smegmatis.

Neutrophils are an important source of IL-12 because they store and produce new IL-12 when they accumulate at sites of infection. The cytokine IL-12 is known to activate and differentiate T cells into TH1 cells, which release IFN- $\gamma$, an activator for bacterial killing in macrophages. Therefore, the TH1 response is typically associated with the clearing of intracellular pathogens like mycobacteria. However, when subjected to both opsonized and non-opsonized M. smegmatis alone, the neutrophils' intracellular IL-12 supply was significantly reduced when compared to untreated cells, and there was no release of the cytokine (Fig. 9A). M. smegmatis reduction of IL-12 levels within the cell provides a mechanism for longterm survival of the bacterium. If IL-12 is not secreted, then T cells and macrophages will remain inactivated, allowing for increased bacterial proliferation and a higher probability of a chronic infection. LPS and IFN- $\gamma$-activated neutrophils simulate the conditions of an ongoing inflammation, and after neutrophils were stimulated with these agonists, neither opsonized nor non-opsonized M. smegmatis was able to make a difference in IL-12 extracellular release when compared to LPS and IFN- $\gamma$ stimulation alone. Thus, M. smegmatis is independently capable of delaying the onset of IL-12 derived inflammatory responses, but once inflammation is ongoing, it has little effect on the neutrophil IL-12 response.

Parenthetically, the LAM subunit of mycobacteria is a known potent inducer of IL-12 release in macrophages (17), but a study between neutrophils and the phosphoinositol-capped version of LAM has never been performed. Despite the decreasing trend seen with the addition of $20 \mu \mathrm{g} / \mathrm{mL}$ of PILAM, it was not statistically significant, indicating that PILAM has no effect on neutrophil release of IL-12 (Fig. 9B). This suggests that PILAM is not involved in IL-12 extracellular release. Although, this could be the case, it 
would be worthwhile to repeat this experiment and obtain more than 4 independent experiments as well to test with higher concentration of PILAM $(40 \mu \mathrm{g} / \mathrm{mL})$ to further confirm these results. In addition to this, it would be interesting to collect the lysates as well as the supernatants, as was done with $M$. smegmatis, to determine if PILAM plays a part in the effect seen with the whole bacterium.

\section{BACTERIAL KILLING}

\section{Neutrophils may or may not kill $M$. smegmatis at $\mathbf{3 0}$ minutes post-infection}
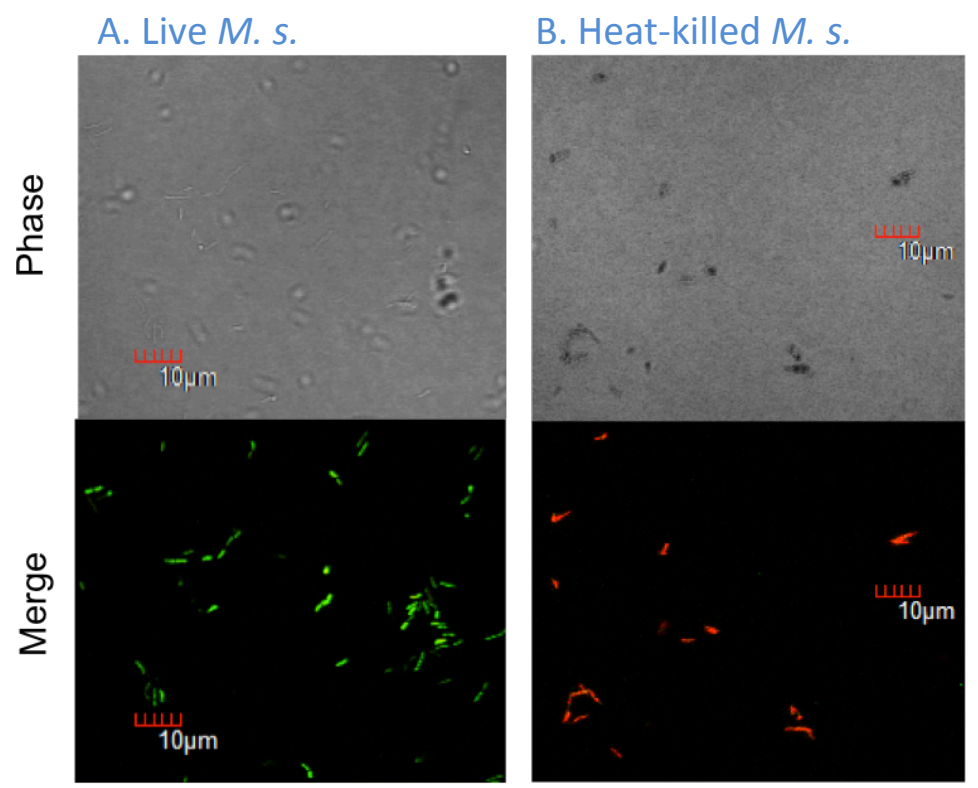

C. UV-killed M.s.

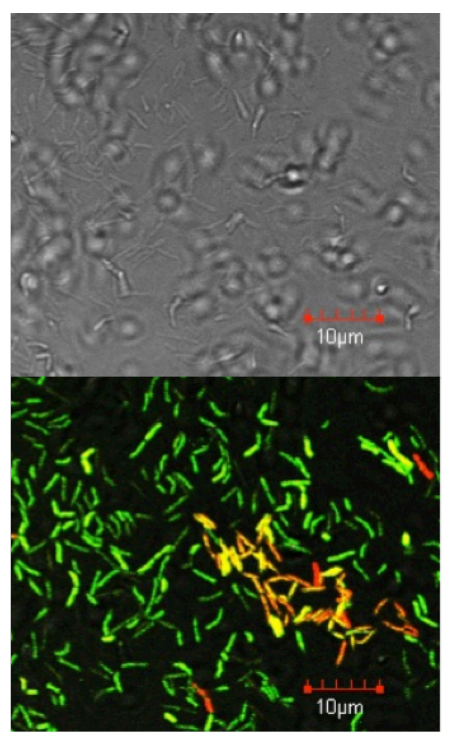

D. Untreated PMN

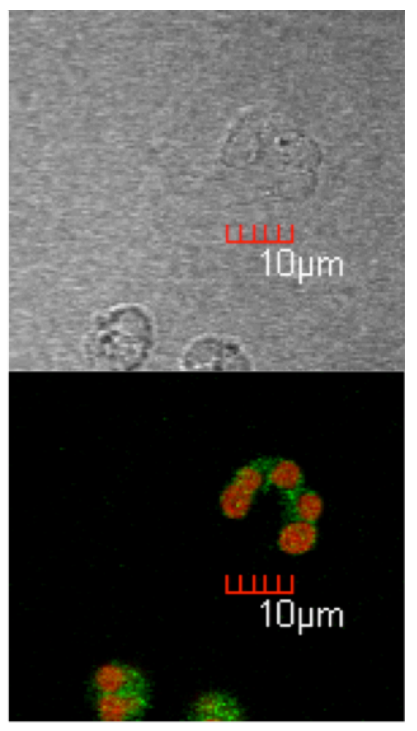

Opsonized M. smegmatis

E. Experiment 1

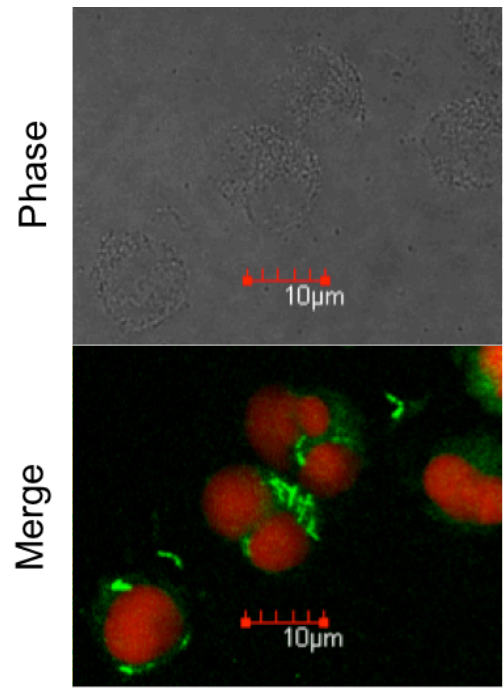

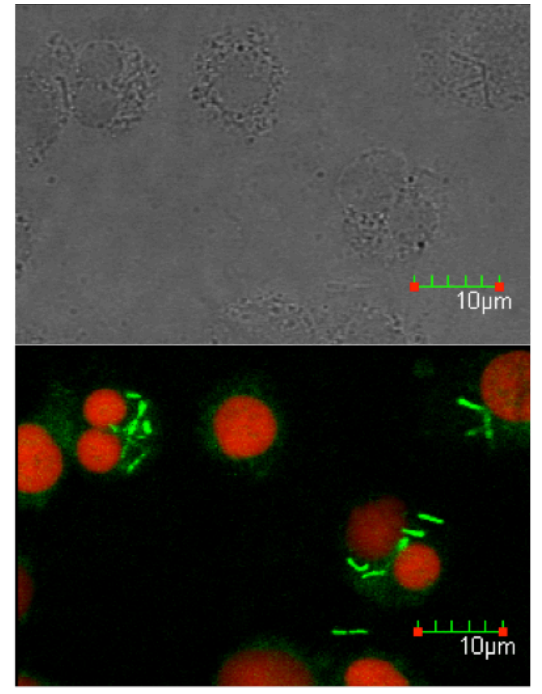

F. Experiment 2
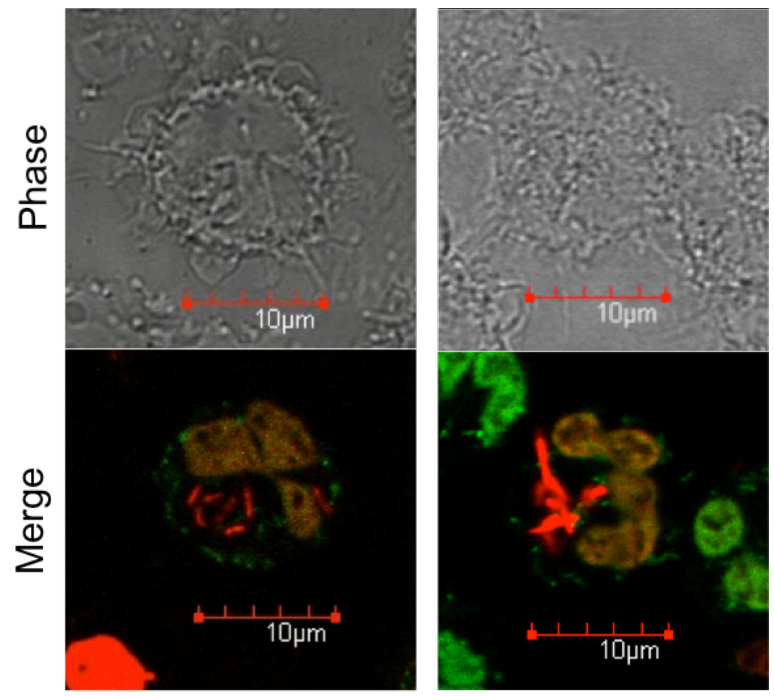

Fig.8 Bacterial viability Assay. Viable M. smegmatis (A), Heat-killed M. smegmatis (B), UV-killed M. smegmatis (C) were treated with the combination of dyes, $30 \mu \mathrm{M}$ PI and $2 \mu \mathrm{M}$ SYTO 9 to use as controls. Then, neutrophils $\left(2 \times 10^{6} \mathrm{cell} / \mathrm{ml}\right)$ were unstimulated (UT) (D), or stimulated with opsonized M. smegmatis (Ms; MOI 6:1 (E, F), in the presence of the PI and SYTO9. Two independent experiments showed that after 30 minutes, intracellular bacteria were either live (E) or unviable (F). 
Finally, an assay measuring bacterial viability was customized to answer the question of how quickly neutrophils kill M. smegmatis. This assay is still under development, which is why there are no definite conclusions for this set of experiments; however, the troubleshooting of the assay has proven to be a valuable source of learning and growth as a scientist.

At first, independent UV-killed and live bacteria conditions were used as controls; the nonviable, UV-killed bacteria would stain red and live bacteria would stain green. However, the UV-killed bacteria, which should have stained 95\% red, were staining a mix of red and green (Fig $8 \mathrm{C}$ ). This was due to the fact that UV exposure makes cells inviable by disrupting their DNA, whereas PI, the dye that labels the dead bacteria, requires a disrupted membrane to enter the cell. As an alternative, heat was used for membrane disruption and killing of M. smegmatis, which resulted in nonviable bacteria staining red and the live, viable bacteria staining a district green (Fig. 8 A, B). The bacteria alone conditions were used to set the laser levels for optimum bacterial visualization inside neutrophils.

The first experiment was a 30-minute challenge that displayed an overwhelmingly green bacterial population indicative of 100\% bacterial survival inside neutrophils (Fig 7 E). This finding was surprising given that our data showed that the presence of $M$. smegmatis incites a robust intracellular respiratory burst at 30 minutes. Contrastingly, when an identical experiment was performed at a later date, the intracellular bacterial population was an overpoweringly red color (Fig. 8 F). In this second experiment, the neutrophils were able to kill almost $100 \%$ of the bacteria internalized, yielding opposite results from the previous experiment. There are several possibilities for the discrepancy of results. First of all, the probability of a technical error cannot be discounted because this is a developing technique. If the laser levels are not at the ideal setting or if the focus is unadjusted, the bacteria may not be visualized. An alternative hypothesis to explain the results is based on the observation that in the all-dead experiment, the Syto9 dyes stained very lightly, giving rise to the chance that the bacterial cells looked as if they were all stained red when some may have actually been alive. The goal is to perform multiple experiments in the future to standardize the data, but to further confirm the ability of neutrophils to kill M. smegmatis another bacteria survival method will be used. Neutrophils will be challenged with opsonized bacteria, and after the 30 min incubation, extracellular bacteria will be removed by antibiotic treatment, following which cells will be lysed and the lysate used to streak a plate, after 2-3 days, the bacterial colony-forming units (CFU) will be counted and compared to bacteria alone condition. If no colonies arise, it will indicate that the bacteria were effectively killed by the neutrophils after 30 min incubation.

If later experiments and colony formation confirm that neutrophils do not kill M. smegmatis within the first 30 minutes after infection, a previous study with $M$. smegmatis and neutrophils could give a possible explanation for this phenomenon. While using an MOI of 50, a group of researchers from France 
found that non-opsonized, un-clumped M. smegmatis impedes the fusion of azurophilic granules with the phagosome (3). They concluded that mycobacteria do not actively control this response, and the effect was mediated through receptors that despite triggering phagocytosis, do not initiate the intracellular signals for fusion of the azurophil granules (3). Nonetheless, in order to mount the most efficient attack, it could be necessary to combine intracellular oxidative burst and granule proteins in the phagosome. If the granule containing the most microbicidal contents is blocked from fusing with the phagosome, the ROS in the phagosome may take longer to kill the bacteria, as was shown in a prior study that compared azurophilic versus specific granule contents' killing efficiency (33). In fact, azurophil granules are so effective at microbial killing that when they were added to macrophages with phagocytized mycobacteria, 85\% of intracellular M. smegmatis were killed within 6 hours post infection (33). Therefore, by avoiding primary granule merging with the phagosome, M. smegmatis is able to prolong its survival and proliferation time intracellularly.

However, it was also found that opsonizing the bacteria with IgG-containing immunoserum could restore the phagosome-azurophil fusion ability (3). The recognition of this antibody could lead to the special uptake of the bacterium through a different set of receptors. Subsequently, the phagocytosis by these new receptors would activate the pathway that is most effective at clearing microbes by combining ROS production and granule fusion with the phagosome. In our study, M. smegmatis was opsonized in pooled human serum which resulted in opsonization by fixing complement but not immunoglobulins. If the optimal way of eliminating intracellular M. smegmatis is through the pooled efforts of oxidative species and granule proteins, the effect caused by the lack of IgG serum opsonization could be one way to explain the majority of live bacteria. However, due to the rarity of $M$. smegmatis infections, it seems unlikely that this mechanism could ward off neutrophil defenses for a prolonged period of time.

\section{Conclusions \& Future Directions}

It had been previously shown that M. smegmatis has the ability to modulate some responses in professional phagocytic cells of the innate immune system like macrophages and neutrophils (3). However, the mechanism for this modulation is unknown and many of the neutrophil bactericidal functions against mycobacteria are still understudied. The goal of this thesis was to make a comprehensive, comparative analysis between neutrophils and different types of $M$. smegmatis treatments (opsonized, non-opsonized) or the PILAM glycolipid that is typically associated with mycobacterial virulence and immune system manipulation. Specifically, degranulation, intra and extracellular ROS production, and cytokine release were examined because mycobacteria has been 
known to proliferate within leukocytes by inhibiting the fusion of the phagosome and lysosomes, decreasing the production of ROS and interfering with the overall activation of the immune cells (33).

Our data showed that either treatment of M. smegmatis or PILAM alone was able to induce only gelatinase granule exocytosis but no induction or reduction of the other three granule subtype was observed. This is highly significant because there has been data to propose that neutrophils kill mycobacteria through oxidation-independent methods (34). Gelatinase granules mainly contain matrix metalloproteases which when release to the extracellular space will induce tissue damage. The ability of M. smegmatis to induce release of gelatinase granule might contribute to tissue damage seen with some M. smegmatis infections.

A different story was discovered when neutrophils were pre-treated with M. smegmatis, followed by activation by fMLF. Pre-treatment with opsonized M. smegmatis resulted in a robust increase of fMLFstimulated secretory vesicle exocytosis, which decline with longer bacteria incubation times. These data suggest that opsonized $M$. smegmatis may act as a priming agent. The decrease on secretory vesicle exocytosis observed with longer bacteria incubations time could be due to stimulation of endocytic events which result in downregulation on the expression of the fMLF receptor. Pre-treatment of neutrophils with either opsonized or non-opsonized M. smegmatis resulted in a modest increase of fMLFstimulated specific granule release. However, pre-treatment with non-opsonized M. smegmatis significantly induced fMLF-stimulated gelatinase and azurophil granule release. This confirmed preceding findings that $M$. smegmatis is unable to independently mobilize the most bactericidal azurophil and specific granules independently, but that it is able to amplify the response of neutrophils when other infectious agents are present (8). Neutrophil priming results in a more severe response against subsequently encountered microbes. In a pro-inflammatory environment that simulates multi-bacterial conditions, the extracellular release of metalloproteases and myeloperoxidase from neutrophil granules could contribute to the host tissue destruction associated with the rare chronic inflammations caused by M. smegmatis.

Similarly, UV-killed bacteria followed the same trend, suggesting that M. smegmatis has no active control on the exocytosis of the microbicidal proteins, but that it is mediated through the signaling pathways triggered by bacterial cell wall components. Consequently, PILAM was tested and found to inhibit fMLF-stimulated azurophil granule release. This finding suggests a mechanism by which mycobacteria could avoid killing by the azurophil granule, although other cell membrane proteins may overpower this effect since the opposite effect was seen with M. smegmatis. It would be interesting to test other infamous proteins from the mycobacterial cell membrane to determine the cause of the effects seen with the entire bacterium. Likewise, using UV-killed M. smegmatis against all the granule types could also 
yield interesting results regarding the level of active modulation of these immune responses by $M$. smegmatis. Another lead to follow in the study of these interactions is to see the amount of degranulation into phagosomes when bacteria are internalized by neutrophils, which was not studied here.

The second major weapon in neutrophils' bactericidal arsenal is the production and release of reactive oxidative species into the extracellular space or into bacteria-containing phagosomes in the cytoplasm. Extracellularly, opsonized M. smegmatis was capable of inciting a time-dependent, extremely robust oxidant production at 60 minutes, further even than the already potent inducer of ROS, S. aureus. This implicates $M$. smegmatis even more as a modulator of innate immune responses because this mechanism is likely associated with its killing, but in the are cases of infection, this response could simultaneously harm host tissues. The result that opsonized M. smegmatis increases extracellular oxidant production contradicts one previous study that found that at an MOI of 50, neither opsonized nor unopsonized bacteria were qualified to augment superoxide production (8). However, compared to the MOI of 6 utilized in this thesis, the discrepancy in responses could be due to the extremely high multiplicity of infection of the prior study. When neutrophils are exposed to such a high amount of bacteria, different pathways and neutrophil functions like apoptosis could be initiated instead, thus yielding the low oxidative response.

Similarly, in a time-dependent fashion, opsonized M. smegmatis increased intracellular oxidant production, more so than non-opsonized M. smegmatis and both opsonized and non-opsonized S. aureus. When compared to the non-opsonized M. smegmatis and S. aureus, this finding emphasized that the uptake method by the neutrophil influences the fate of the microbe. Opsonization leads to recognition and phagocytosis through the complement and/or Fc $\gamma \mathrm{R}$ receptor, which activates a different pathway, leading to a more exaggerated oxidant production. The difference between the opsonized M. smegmatis and S. aureus may be due to PILAM's influence. Not only was PILAM able to significantly induce intracellular ROS, but also, it was able to prime the intracellular response of S. aureus-induced ROS. However, despite the amplified intracellular oxidant response in regards to M. smegmatis, it was unknown whether it occurred at the phagosome where pathogenic mycobacteria are known to proliferate. Additionally, other sources have proposed that M. smegmatis is among the ranks of mycobacteria that are able to delay phagosome maturation and acidification by blocking the recruitment of lysosomes to the vesicle in macrophages $(35,36)$. Nonetheless, images taken using a confocal microscope confirm that the intracellular ROS is taking place at the phagosome, where the bacteria are localized. What remains unknown is whether or not there is phagosome maturation involving the granules, which points to a direction this study may explore in the future. 
Another major function of neutrophils explored in this study was the release of cytokines that mediate the transition between the innate to adaptive immunity, notably IL-12. Neutrophils have recently become recognized as an important source for this molecule, which leads to favorable responses against intracellular pathogens during the initial stages on infection. Neutrophil challenge with both opsonized and non-opsonized $M$. smegmatis led to the decrease in intracellular storage of IL-12, as compared to the untreated cells. This interference in neutrophils' IL-12 production and storage could sway immune responses, allowing $M$. smegmatis or other bacteria present to continue surviving and proliferating. Lastly, a technique for determining M. smegmatis'viability post-infection is being developed, although definitive results have not been acquired. The two experiments performed had opposite results, one displaying minimal microbial killing after a 30 minute challenge, and the other showing almost complete annihilation of the internalized bacteria. For the presented studies primary human neutrophils from healthy donors are used and there is variation between individuals which could explain the different results. Even though the experiment requires more attempts in order to standardize the data, previous studies with $M$. smegmatis give plausible explanations for either outcome. It has been propositioned that M. smegmatis has the capability of blocking the fusion of the phagosome with azurophil granules, which could delay the killing of the microbe (3). Likewise, M. smegmatis contains an oxidant scavenger protein, SOD, which could alleviate the oxidative stress from the respiratory burst. These mechanisms could be collaborating to keep the bacteria alive for as long as possible. In addition to visual inspection via confocal microscopy, future studies will utilize another technique to evaluate bacteria viability by counting colony-forming units

In conclusion, neutrophils' responses to M. smegmatis indicate that this non-pathogenic, environmental mycobacterium can promote inflammation, tissue damage, and manipulate the $\mathrm{T}_{\mathrm{h}} 1$ response by controlling production of IL-12. Also, our data suggests that PILAM is involved in the $M$. smegmatis modulation of intracellular ROS, gelatinase granule exocytosis and IL-12 release. Therefore, the interaction between these avirulent bacteria and human neutrophils is more complex and interesting than ever before. 


\section{Acknowledgements}

I would like to especially thank Dr. Silvia Uriarte and Dr. James Graham for their support as my mentors, Charles Anderson and Lee Cahill for growing M. smegmatis for this study, Junyi Le and Cortney Armstrong for their technical assistance, Terri Manning for obtaining donors and isolating the neutrophils, and Michael Creed for his assistance with the statistical analyses. 


\section{Works cited}

1. Janeway CA Jr, T. P., Walport M, et al. 2001. Principles of innate and adaptive immunity. In Immunobiology: The Immune System in Health and Disease. Garland Science, New York.

2. $\quad$ Borregaard, N. 2010. Neutrophils, from marrow to microbes. Immunity 33: 657-670.

3. Cougoule, C., P. Constant, G. Etienne, M. Daffe, and I. Maridonneau-Parini. 2002. Lack of fusion of azurophil granules with phagosomes during phagocytosis of Mycobacterium smegmatis by human neutrophils is not actively controlled by the bacterium. Infection and immunity 70: 1591-1598.

4. Nauseef, W. M. 2007. How human neutrophils kill and degrade microbes: an integrated view. Immunological Reviews 219: 88-102.

5. Mocsai, A. 2013. Diverse novel functions of neutrophils in immunity, inflammation, and beyond. The Journal of experimental medicine 210: 1283-1299.

6. Nordenfelt, P., and H. Tapper. 2011. Phagosome dynamics during phagocytosis by neutrophils. Journal of leukocyte biology 90: 271-284.

7. Lowe, D. M., P. S. Redford, R. J. Wilkinson, A. O'Garra, and A. R. Martineau. 2012. Neutrophils in tuberculosis: friend or foe? Trends Immunol 33: 14-25.

8. N'Diaye, E. N., X. Darzacq, C. Astarie-Dequeker, M. Daffe, J. Calafat, and I. Maridonneau-Parini. 1998. Fusion of azurophil granules with phagosomes and activation of the tyrosine kinase Hck are specifically inhibited during phagocytosis of mycobacteria by human neutrophils. Journal of immunology 161: 4983-4991.

9. Borregaard, N., O. E. Sørensen, and K. Theilgaard-Mönch. 2007. Neutrophil granules: a library of innate immunity proteins. Trends in Immunology 28: 340-345.

10. Borregaard, N., and J. B. Cowland. 1997. Granules of the human neutrophilic polymorphonuclear leukocyte. Blood 89: 3503-3521.

11. Dahlgren, C., and A. Karlsson. 1999. Respiratory burst in human neutrophils. Journal of immunological methods 232: 3-14.

12. Levay, P. F., and M. Viljoen. 1995. Lactoferrin: a general review. Haematologica 80: 252-267.

13. Mark B. Hampton, A. J. K. a. C. C. W. 1998. Inside the Neutrophil Phagosome: Oxidants, Myeloperoxidase, and Bacterial Killing. Blood 92: 3007-3017.

14. Ganz, T. 2003. Defensins: antimicrobial peptides of innate immunity. Nature reviews. Immunology 3: 710-720.

15. Mantovani, A., M. A. Cassatella, C. Costantini, and S. Jaillon. 2011. Neutrophils in the activation and regulation of innate and adaptive immunity. Nature reviews. Immunology 11: 519-531.

16. Futosi, K., S. Fodor, and A. Mocsai. 2013. Neutrophil cell surface receptors and their intracellular signal transduction pathways. International immunopharmacology 17: 638-650.

17. Dao, D. N., L. Kremer, Y. Guerardel, A. Molano, W. R. Jacobs, Jr., S. A. Porcelli, and V. Briken. 2004. Mycobacterium tuberculosis lipomannan induces apoptosis and interleukin-12 production in macrophages. Infection and immunity 72: 2067-2074. 
18. Berger, A. 2000. Th1 and Th2 responses: what are they? BMJ (Clinical research ed.) 321: 424.

19. Ellis, T. N., and B. L. Beaman. 2004. Interferon-gamma activation of polymorphonuclear neutrophil function. Immunology 112: 2-12.

20. D'Elios, M. M., M. Benagiano, C. Della Bella, and A. Amedei. 2011. T-cell response to bacterial agents. Journal of infection in developing countries 5: 640-645.

21. Shimizu, F. 2012. Mycobacterium smegmatis soft tissue infection. International Journal of Dermatology 51: 1518-1520.

22. Primm, T. P., C. A. Lucero, and J. O. Falkinham, 3rd. 2004. Health impacts of environmental mycobacteria. Clinical microbiology reviews 17: 98-106.

23. Pelosi, A., D. Smith, R. Brammananth, A. Topolska, H. Billman-Jacobe, P. Nagley, P. K. Crellin, and R. L. Coppel. 2012. Identification of a Novel Gene Product That Promotes Survival of $<$ italic $>$ Mycobacterium smegmatis $<$ /italic $>$ in Macrophages. PLoS ONE 7: e31788.

24. Anes, E., P. Peyron, L. Staali, L. Jordao, M. G. Gutierrez, H. Kress, M. Hagedorn, I. Maridonneau-Parini, M. A. Skinner, A. G. Wildeman, S. A. Kalamidas, M. Kuehnel, and G. Griffiths. 2006. Dynamic life and death interactions between Mycobacterium smegmatis and J774 macrophages. Cellular microbiology 8: 939-960.

25. Kumar, K. J., J. Chandra, R. N. Mandal, R. Dutta, and N. K. Jain. 1995. Fatal pulmonary infection caused by Mycobacterium smegmetis in an infant. Indian journal of pediatrics 62: 619-621.

26. Chadha, V. K. 2001. Tuberculin test. Indian journal of pediatrics 68: 53-58.

27. Vukmanovic-Stejic, M., J. R. Reed, K. E. Lacy, M. H. A. Rustin, and A. N. Akbar. 2006. Mantoux Test as a model for a secondary immune response in humans. Immunology letters 107: 93-101.

28. Faldt, J., C. Dahlgren, M. Ridell, and A. Karlsson. 2001. Priming of human neutrophils by mycobacterial lipoarabinomannans: role of granule mobilisation. Microbes and infection / Institut Pasteur 3: 1101-1109.

29. Briken, V., S. A. Porcelli, G. S. Besra, and L. Kremer. 2004. Mycobacterial lipoarabinomannan and related lipoglycans: from biogenesis to modulation of the immune response. Molecular microbiology 53: 391-403.

30. Shiloh, M. U., and P. A. Champion. 2010. To catch a killer. What can mycobacterial models teach us about Mycobacterium tuberculosis pathogenesis? Current opinion in microbiology 13: 86-92.

31. Uriarte, S. M., M. J. Rane, G. C. Luerman, M. T. Barati, R. A. Ward, W. M. Nauseef, and K. R. McLeish. 2011. Granule exocytosis contributes to priming and activation of the human neutrophil respiratory burst. Journal of immunology 187: 391-400.

32. Johnson, M. B., and A. K. Criss. 2013. Fluorescence microscopy methods for determining the viability of bacteria in association with mammalian cells. Journal of visualized experiments : JoVE.

33. Jena, P., S. Mohanty, T. Mohanty, S. Kallert, M. Morgelin, T. Lindstrom, N. Borregaard, S. Stenger, A. Sonawane, and O. E. Sorensen. 2012. Azurophil granule proteins constitute the major mycobactericidal proteins in human neutrophils and enhance the killing of mycobacteria in macrophages. PLoS One 7: e50345. 
34. Jones, G. S., H. J. Amirault, and B. R. Andersen. 1990. Killing of Mycobacterium tuberculosis by neutrophils: a nonoxidative process. The Journal of infectious diseases 162: 700-704.

35. Mishra, A. K., N. N. Driessen, B. J. Appelmelk, and G. S. Besra. 2011. Lipoarabinomannan and related glycoconjugates: structure, biogenesis and role in Mycobacterium tuberculosis physiology and host-pathogen interaction. FEMS microbiology reviews 35: 1126-1157.

36. Pelosi, A., D. Smith, R. Brammananth, A. Topolska, H. Billman-Jacobe, P. Nagley, P. K. Crellin, and R. L. Coppel. 2012. Identification of a novel gene product that promotes survival of Mycobacterium smegmatis in macrophages. PLoS One 7: e31788. 